\title{
Polycystin-1 maturation requires polycystin-2 in a dose-dependent manner
}

\author{
Vladimir G. Gainullin, ${ }^{1}$ Katharina Hopp, ${ }^{2}$ Christopher J. Ward, ${ }^{2}$ Cynthia J. Hommerding, ${ }^{2}$ and Peter C. Harris ${ }^{1,2}$ \\ DDepartment of Biochemistry and Molecular Biology and 2Division of Nephrology and Hypertension, Mayo Clinic, Rochester, Minnesota, USA.
}

\begin{abstract}
Autosomal dominant polycystic kidney disease (ADPKD) is a common inherited nephropathy responsible for $4 \%-10 \%$ of endstage renal disease cases. Mutations in the genes encoding polycystin-1 (PC1, PKD1) or polycystin-2 (PC2, PKD2) cause ADPKD, and PKD1 mutations are associated with more severe renal disease. PC1 has been shown to form a complex with PC2, and the severity of PKD1-mediated disease is associated with the level of the mature PC1 glycoform. Here, we demonstrated that PC1 and PC2 first interact in the ER before PC1 cleavage at the CPS/CAIN site and determined that PC2 acts as an essential chaperone for PC1 maturation and surface localization. The chaperone function of PC2 was dependent on the presence of the distal coiled-coil domain and was disrupted by pathogenic missense mutations. In Pkd2-/- mice, complete loss of PC2 prevented PC1 maturation. In Pkd2 heterozygotes, the 50\% PC2 reduction resulted in a nonequimolar reduction (20\%-25\%) of the mature PC1 glycoform. Interbreeding between various $P k d 1$ and $P k d 2$ models revealed that animals with reduced levels of functional PC1 and PC2 in the kidney exhibited severe, rapidly progressive disease, illustrating the importance of complexing of these proteins for function. Our results indicate that PC2 regulates PC1 maturation; therefore, mature PC1 levels are a determinant of disease severity in PKD2 as well as PKD1.
\end{abstract}

\section{Introduction}

Autosomal dominant polycystic kidney disease (ADPKD) is a common (frequency 1:600-1:1,000), progressive nephropathy accounting for $4 \%-10 \%$ of patients with end-stage renal disease (ESRD) (1). Mutations to PKD1 ( 85\% of cases) or PKD2 ( 15\%) cause clinically indistinguishable ADPKD, except that PKD2 patients typically experience ESRD more than 20 years later than PKD1 patients with fully inactivating mutations (79.7 years vs. 55.6 years) (2-5). PKD2 encodes polycystin-2 (PC2), a 968-aa, 6-transmembrane protein of the TRPP family of calcium-regulated cation channels $(2,6,7)$. Polycystin-1 (PC1), encoded by PKD1, is a large glycoprotein (4,303 aa) with an approximately 3,000-aa N-terminal ectodomain, anchored to the membrane by 11 transmembrane helices and with a short C-terminal cytoplasmic tail (3). PC1 undergoes cis-autoproteolytic cleavage at the conserved GPS/GAIN site immediately preceding the first transmembrane helix, a feature of many adhesion-type $G$ protein-coupled receptors (e.g., latrophilin) $(8-10)$. The 2 cleaved products remain attached, and missense mutations within the approximately 320-aa GPS/GAIN domain block the severing $(10,11)$. Rapidly progressive PKD develops when cleavage is blocked (12), underscoring the importance of this region for PC1 function.

Several subcellular localizations have been suggested for the polycystins, including cilia, plasma membrane (PM), exosomelike vesicles (ELVs), and endoplasmic reticulum (ER) (13-19). The existence of 2 different GPS-cleaved, N-terminal PC1 glycoforms also supports different subcellular localizations $(14,20)$. Evidence from PC homologs and syndromic forms of PKD sug-

Conflict of interest: The authors have declared that no conflict of interest exists. Submitted: May 19, 2014; Accepted: December 2, 2014.

Reference information: J Clin Invest. 2015;125(2):607-620. doi:10.1172/JCI76972. gests that the primary cilium is central to pathogenesis (21-25); ciliary locations are described for both PC proteins $(26,27)$. Nevertheless, the functional site of the polycystins for cystogenesis remains controversial (28-30).

Consistent with the similar PKD1/PKD2 phenotypes, exogenous expression of PC1 and PC2 constructs and endogenous analysis show interactions between the coiled-coil domains in the C-terminal tails of the proteins $(15,18,31,32)$. Recent data indicated that a newly defined coiled coil (CC2) at the distal end of PC2 binds PC1 $(33,34)$. Although PC2 binding has been suggested to influence PC1 GPS/GAIN cleavage, as has the PC1 C-terminal tail $(15,17)$, limited data are available about the significance of PC1 and PC2 complexing for protein maturation, especially analyzing endogenous proteins.

PC2 has been described as influencing PC1 trafficking $(15,17)$, although PC2 may also move independently of PC1 to the cilium $(19,35)$. PC1 has been proposed to be the regulatory subunit of a heteromeric complex with PC2 (although the precise stoichiometry of the complex is uncertain), mediating a mechanosensorystimulated $\mathrm{Ca}^{2+}$ influx $(15,23,36,37)$. PC2 can also complex with other TRP channels $(38,39)$, and PC2 partners with another polycystin, PC1L1, in the node (40-42). Hence, questions remain about the independence of PC1 and PC2, and the importance of the partner protein for appropriate localization.

Homozygous Pkd1- and Pkd2-null mice have embryoniclethal phenotypes ( $\sim$ E14.5), with indistinguishable renal cystic disease, while heterozygotes develop few cysts (43-45). Bilineal mice $\left(P k d 1^{+-} P k d 2^{+-}\right)$develop few cysts, but more than expected from a simple additive effect (46). In the 1 described bilineal PKD1 and $P K D 2$ family, the $2 P K D 1^{+/-} P K D 2^{+/-}$patients developed ESRD approximately 15 years earlier than other PKD1 or PKD2 family members, indicating a combined contribution to the phenotype 
A

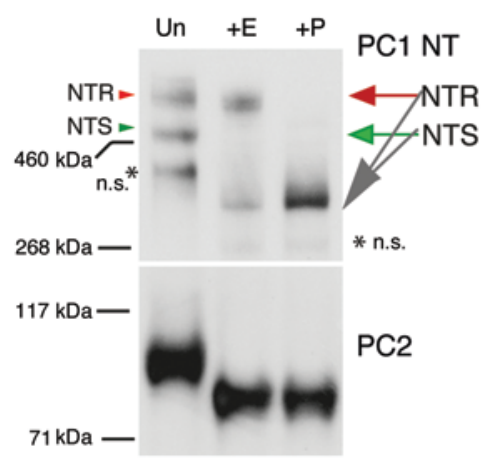

B
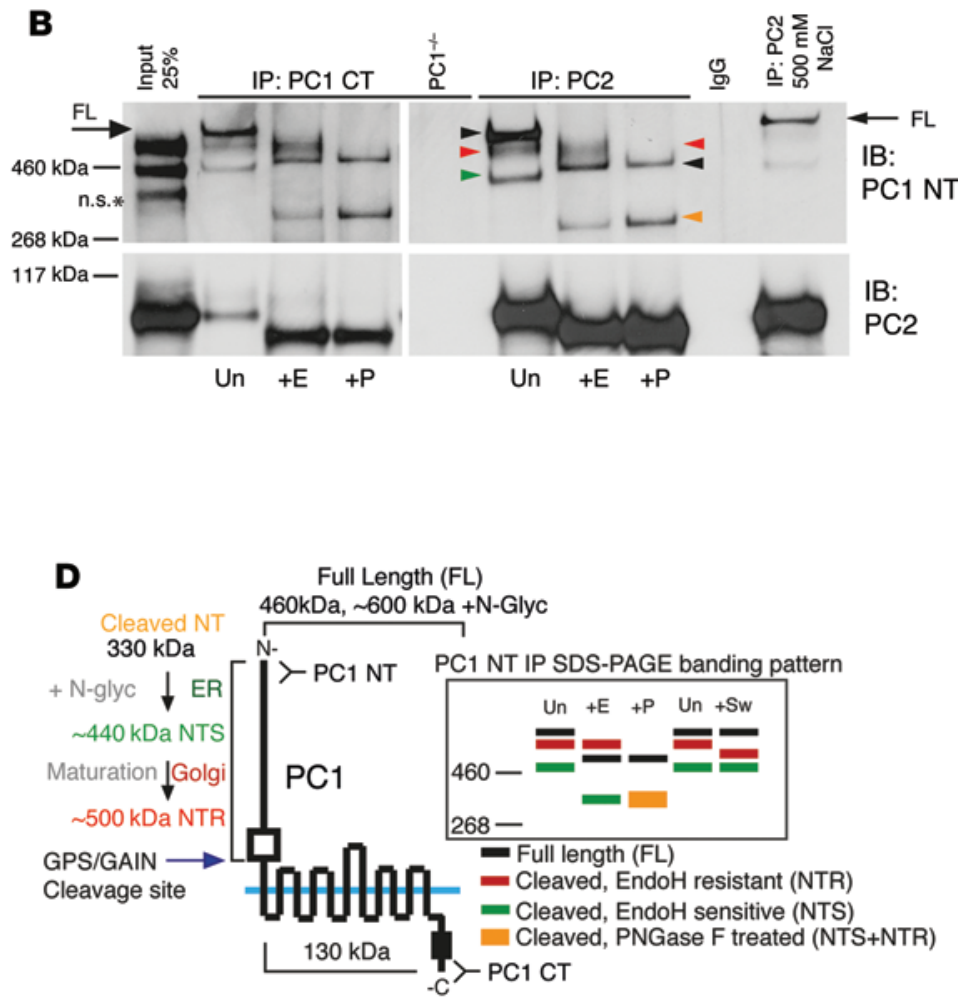

Figure 1. Processing, complexing, and localization of PC1 and PC2. (A) Immunoblot (IB) of endogenous human PC1 and PC2 derived from membrane fractions of a renal cortical tubule epithelial (RCTE) cell line. Samples were untreated (Un) or treated with EndoH (+E) or PNCase $F(+P)$ and detected with an antibody against N-terminal PC1 (7e12; PC1 NT) or PC2 (YCE2). A nonspecific protein (Supplemental Figure 1, A and B) is indicated (n.s.). N-terminal glycoproducts, EndoH resistant (NTR) and EndoH sensitive (NTS), were resolved and were both reduced to the size of the aa backbone with PNCase $\mathrm{F}$ treatment ( $330 \mathrm{kDa})$. All of PC2 was sensitive to EndoH. Representative blots are shown from 3 independent experiments. (B) IPs with a PC1 CT (BD3) or PC2 (YCE2) antibody from RCTE cells followed by deglycosylation detected with PC1 NT or YCE2 (PC2). PKD1 ${ }^{-/-}$epithelial cells (9-12 cells; PKD1 ${ }^{-/-}$) and IP with irrelevant antibody (IgG) were used as negative controls. The PC1 and PC2 complex was formed in the ER (EndoH sensitive), since PC2 coimmunoprecipitated all PC1 glycoforms, including PC1-FL, even in high-salt (500 mM NaCl) conditions. Representative blots are shown from 3 independent experiments. (C) Maturation of PC1-NTR was affected by $2 \mu \mathrm{g} / \mathrm{ml}$ swainsonine (+Sw) treatment. A 72-hour swainsonine treatment reduced the PC1-NTR molecular weight but did not affect PC1-NTS, PC1-FL, or PC2, indicating that only PC1, but not PC2, traffics through the Golgi apparatus. Representative blots are shown from 3 independent experiments. (D) Schematic of PC1 cleavage and glycosylation showing the size of the FL and the 2 GPS/CAIN $\mathrm{N}$-terminal cleavage products, NTS and NTR.

$(47,48)$. Cyst development in ADPKD may require somatic second hits. However, other data suggest a threshold/dosage model of cystogenesis in which cysts develop with some polycystin present due to stochastic and other factors, with the PKD severity related to the overall availability of functional polycystin $(20,49,50)$.

Here we evaluate the processing, maturation, and localization of PC1, studying the endogenous protein wherever possible. The role that PC2 plays in this process is our focus, and we conclude that PC2 acts as a critical chaperone for PC1. Additionally, wholeanimal studies reinforce the role of genetic interaction of $P k d 1$ and $P k d 2$ in the cystogenic process. These findings have important implications for understanding the pathogenesis of this disorder and suggest a high level of interplay between the 2 diseases.

\section{Results}

Endogenous PC1-PC2 complex in the ER before GPS/GAIN cleavage of $P C 1$. We initially analyzed the glycosylation pattern of the human polycystin complex in a renal cortical tubule epithelial (RCTE) cell line using peptide- $N$-glycosidase F (PNGase F), to remove all sugars, and endoglycosidase $\mathrm{H}$ (EndoH), to excise high-mannose sug- ars typical of cis-Golgi-localized proteins. Consistent with previous findings from mouse tissue (20), PNGase F digestion showed that nearly all of human PC1 was cleaved at the GPS/GAIN site, and EndoH analysis indicated that approximately $50 \%$ of the PC1 $\mathrm{N}$-terminal (NT) product was resistant (NTR; mature), and so likely surface localized, while the other approximately $50 \%$ was EndoH sensitive, suggesting ER localization (NTS) (Figure 1, A and D, and Supplemental Figure 1, A and B; supplemental material available online with this article; doi:10.1172/JCI76972DS1). All detectable PC2 was EndoH sensitive, consistent with an ER localization (13). Immunoprecipitation (IP) with antibodies to the PC1 C-terminus (CT) or PC2 and detection with the PC1 NT antibody confirmed that the GPS/GAIN-cleaved products remained attached (Figure 1, B and D, Supplemental Figure 1E, and ref. 10). Redetection with the PC1 CT antibody PKS-A (51) identified both the $\mathrm{C}$-terminal EndoH-sensitive (CTS) and C-terminal EndoHresistant (CTR) GPS products (Supplemental Figure 1, C and D). Interestingly, the normally rare full-length (FL; uncleaved) PC1, which was enriched by CT-associated IP (as well as the NT and CT glycoforms), coimmunoprecipitated with PC2, and was espe- 
cially seen under stringent (high-salt) conditions (Figure 1B and Supplemental Figure 1E). This indicates that complexing initiated in the ER before GPS/GAIN cleavage. Prolonged treatment with swainsonine, a small-molecule inhibitor of Golgi $\alpha$-mannosidase II that blocks mature (post-ER) glycosylation (52), affected only PC1-NTR, not PC1-FL, PC1-NTS, or PC2 (Figure 1, C and D, and Supplemental Figure $1 \mathrm{~F}$ ). Combined with the deglycosylation analyses, these data demonstrate that in the ER, PC2 complexes with PC1-FL and PC1-CTS, as well as PC1-NTS via PC1-CTS/PC1NTS tethering, before PC1 maturation. Since PC2 was not affected by swainsonine and remained sensitive to EndoH, these data suggest that only PC1 passes through the Golgi, while PC2 is mostly retained in the ER.

Localization of PC1 glycoforms and PC2. Our results show that both ER and post-Golgi pools of PC1 coimmunoprecipitate with PC2, although PC2 retains ER-specific oligosaccharides. To determine whether mature PC1 and EndoH-sensitive PC2 were PM localized, we specifically labeled sialic acids, a marker of mature glycoproteins (53), on RCTE cell surfaces with $1 \mu \mathrm{M} \mathrm{NaIO}_{4}$ and alkoxyamine biotin, catalyzed by aniline. This surface glycoprotein labeling and IP specifically precipitated mature PC1 (NTR) but not PC1-NTS (Figure 2A). Analysis of PC2 showed that a small fraction of this protein was also precipitated with the surface complex, despite being EndoH sensitive (Figure 2A). To further resolve the site of these endogenous proteins, we analyzed their fractionation pattern on OptiPrep gradients (2\%-30\%). Most detectable PC2 localized to ER enriched fractions (calnexin, STIM1), which was also the location of PC1-NTS (Figure 2B). PC1-NTR was enriched in fractions containing PM proteins (ORAI-1, SMO), which did not seem to contain PC2. PC1-NTR was also found within the primary cilia proteins (Arl13b, SMO) enriched fraction, along with a low level of PC2. We conclude that the initial PC1-PC2 interaction occurs in the ER; however, most PC1 is trafficked alone through the Golgi to the PM. One explanation for the PC1-NTR and PC2 interaction and the surface localization data is that they complex while in different membranes, PC1-NTR in the PM and PC2 in the ER (54), similar to ORAI-1 (PM) and STIM1 (ER) at PM/ER junctions $(55,56)$. Indeed, a small amount of ORAI-1/STIM1 overlapped in fractions 12-14, which also contain both PC1 and PC2, albeit in relatively small amounts. Further analysis of the cilia enriched fraction showed only the PC1-NTR form of PC1 along with the EndoH-sensitive form of PC2 (Figure 2C). This is consistent with independent trafficking (bypassing the Golgi) of an EndoH-sensitive form of PC2 to cilia (19).

To explore the localization of the polycystins by immunofluorescence (IF), we exogenously expressed N-terminally tagged proteins, mCherry-PC1 and GFP-PC2, in the RCTE cell line (Figure $3 \mathrm{~A})$. Surface prefixation labeling of PC1 and confocal analysis with an mCherry antibody confirmed that a fraction of PC1 was PM localized (Figure 3B). Surface PC1 signal occasionally colocalized with PC2 in specific punctae but did not universally overlap, consistent with separate PM and ER localizations (55). Total PC1 signal overlapped with PC2 in the ER (Figure 3B and Supplemental Figure 2, A and C). Localization of surface-labeled and total mCherry-PC1 with GFP-PC2 in RCTE cells induced to develop cilia showed both proteins colocalized on cilia in about $60 \%$ of ciliated cells (Figure 3C and Supplemental Figure 2, B and C). Over- all, our data are consistent with PC1-NTR and PC2 interacting in the ER and across PM/ER junctions, but also show that both proteins are found on primary cilia. In both cases, ER-based PC1-PC2 complexes may disassemble to allow independent trafficking.

Since previous studies suggested that PC2 influences PC1 localization and processing $(15,17)$ and our data show that PC2 interacts with PC1-FL and the ER glycoforms of PC1 (NTS, CTS), we analyzed the role of PC2 in PC1 cleavage and maturation. Consistent with the described enhanced PC1 trafficking with PC2 coexpression (17), analysis of exogenously expressed tagged polycystins showed strong prefixation surface labeling of mCherryPC1 and the presence of the PC1-NTR glycoform only when GFPPC2 was coexpressed (Figure 3, D and E). In the absence of GFPPC2, mCherry-PC1 was not detected on the cell surface but accumulated as its FL and NTS glycoforms (Figure 3E). The absence of detectable PC1-NTR in RCTE cells without GFP-PC2 cotransfection, although they endogenously express PC2 (Figure 1), is likely explained by the large differences in expression levels (approximately tenfold) between endogenous and exogenous PC1 and PC2 (Supplemental Figure 3). At the exposure that only reveals exogenously expressed protein (Figure 3E), PC1-NTR is not evident without coexpression of PC2 at relatively high levels. Taken together, these data indicate that PC2 facilitates ER exit and maturation of PC1.

PC2 is required for endogenous PC1 maturation. Consistent with the exogenous expression data, analysis of mouse embryonic fibroblasts (MEFs) from $P k d 2^{-/}$mice showed that PC1-NTR was completely lost and PC1-NTS increased, sometimes substantially, when PC2 was absent (Figure 4, A and B). An increased level of PC1-FL was also seen in $P k d 2^{-/-}$mice, compared with $\mathrm{Pkd2}^{+--}$and WT mice. Next, we tested the effect of PC2 loss on PC1 secretion. Only the PC1-NTR glycoform has previously been shown secreted on ELVs in urine and from cells in culture $(14,20)$. Analysis of total media protein from cultured MEFs showed no PC1 secreted from $P k d 2^{--}$cells (Supplemental Figure 4); therefore, the absence of PC1-NTR when PC2 is lost is not due to increased secretion of this product. Previous exogenous expression studies suggested that PC2 trafficked to cilia independently of PC1 $(19,35)$; however, when we analyzed $P k d 1^{-/-}$MEFs, PC2 was not detected on cilia (Figure 4, C and D). Although our data demonstrated that endogenous PC1 maturation and, hence, PM and cilia localization require PC2, confirmation of these findings using IF was not possible because of the lack of IF detection of endogenous PC1 with available PC1 antibodies. These data show that endogenous PC1 maturation and secretion require PC2, and that in MEFs PC2 cilia localization depends on PC1.

PC1 maturation is regulated by the dosage of PC2. Next we explored whether there was a corresponding reduction in the level of PC1-NTR if PC2 was reduced but not completely eliminated. Interestingly, compared with the expected $50 \%$ reduction of PC1NTR (and PC1-NTS) observed in $\mathrm{Pkd1}^{+/-} \mathrm{MEFs}, \mathrm{Pkd2}^{+-}$kidneys and MEFs showed a modest ( 25\%) but significant reduction of mature PC1 (NTR) (Figure 4A and Figure 5, A and C). We previously reported in the hypomorphic $P k d 1^{R 3277 C / R 3277 C}\left(P k d 1^{R C / R C}\right)$ mouse model that PC1-NTR in ELVs was reduced to approximately $40 \%$ of WT (20), and quantification here in MEFs showed a consistent decrease to approximately $35 \%$ (Figure $5, A^{-} \mathrm{C}$ ), with 
$\mathbf{A}$
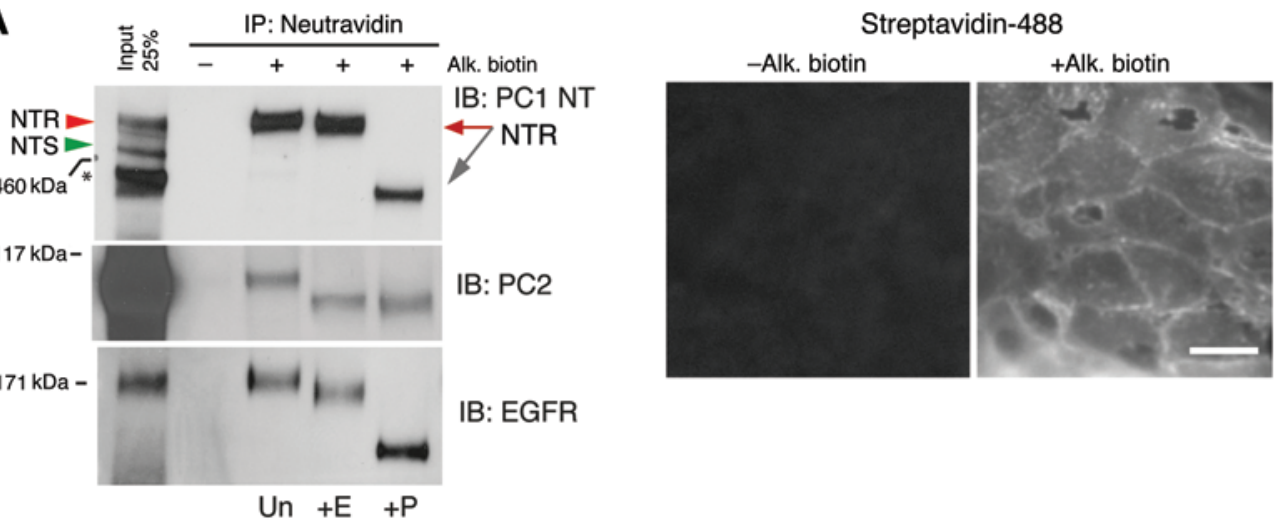

B

OptiPrep gradient (concentration)
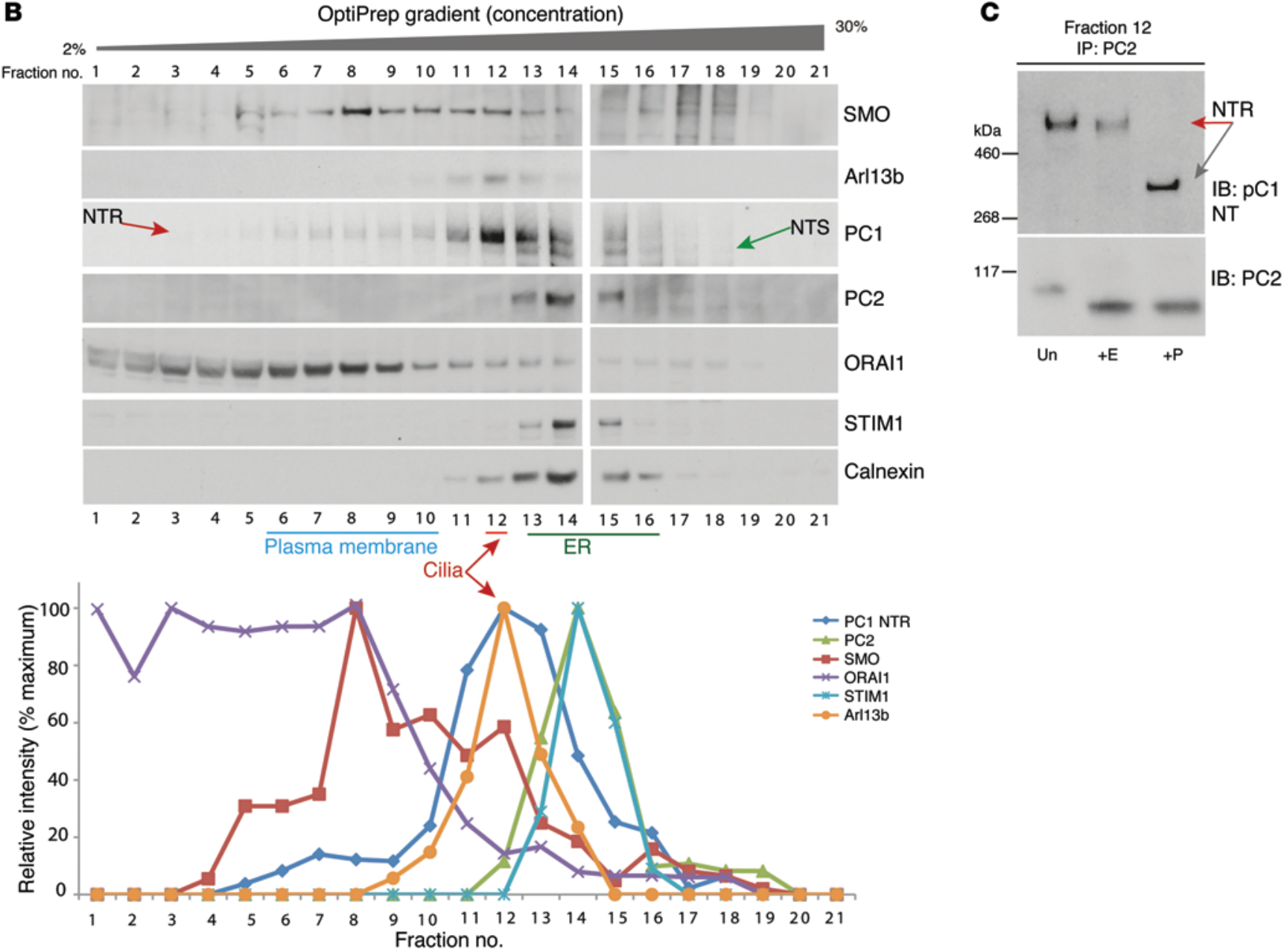

Figure 2. Subcellular localization of PC1 glycoforms. (A) Labeling of RCTE cell surface proteins using alkoxyamine biotin (Alk. Biotin) and IP with neutravidin shows that PC1-NTR was the sole PC1 glycoform localized to the cell surface. PC2 was only detected with neutravidin IP after prolonged exposure and remained sensitive to EndoH digestion; compare level of input and surface protein. EGFR was used as a PM protein control. Representative blots are shown from 3 independent experiments. IF of streptavidin-488-labeled Alk. Biotin-treated and untreated cells shows efficient surface glycoprotein labeling. Scale bar: $20 \mu \mathrm{m}$. (B) Density gradient fractionation of RCTE cells shows that a portion of PC1-NTR cofractionated with markers of PM (ORAI/ SMO) and cilia (Arl13b/SMO), while most detectable PC2 was distributed in fractions overlapping with ER proteins, calnexin, and STIM1, with minor overlap with the cilia fraction. The relative signal intensity is plotted below. Gradient samples were loaded on 2 different SDS-PAGE gels that were run simultaneously and transferred onto the same membrane for detection. Representative blots are shown from 3 independent experiments. (C) Coimmunoprecipitation of PC2 with PC1 from gradient fraction 12 (cilia enriched) followed by deglycosylation using EndoH (+E) or PNGase F (+P) or no enzyme (Un). Only the EndoH-resistant PC1-NTR glycoform along with EndoH-sensitive PC2 cofractionated with Arl13b. 
A

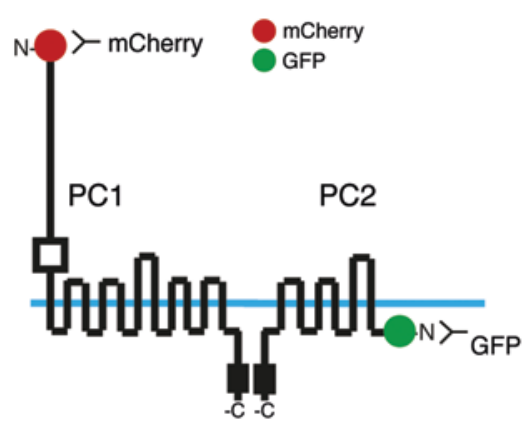

B
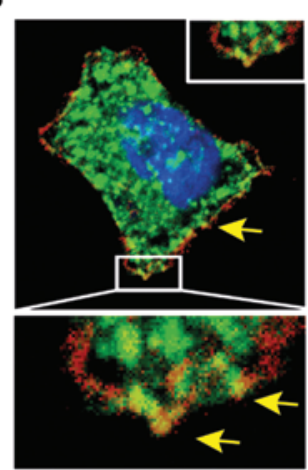

Surface

mCherry-PC1

GFP-PC2 + DAPI
Total

mCherry-PC1
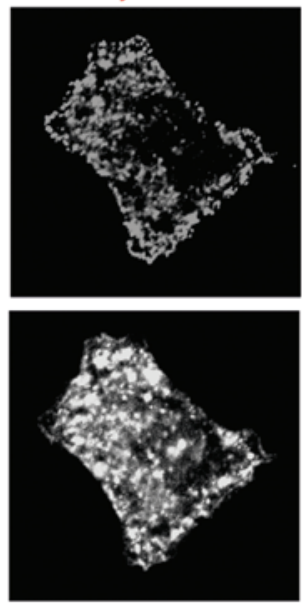

GFP-PC2
Surface

mCherry-PC1
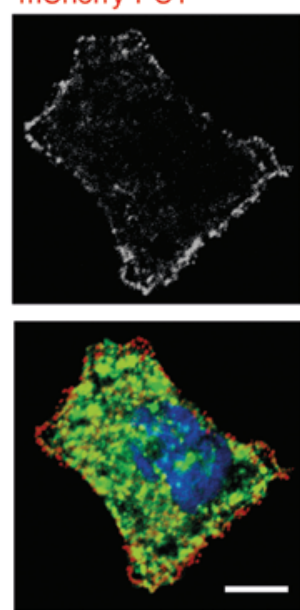

mCherry-PC1 (total) GFP-PC2 + DAPI
C Surface

mCherry-PC1

GFP-PC2 + DAPI

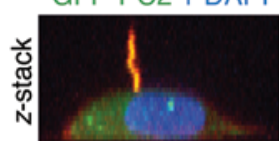

Surface

mCherry-PC1
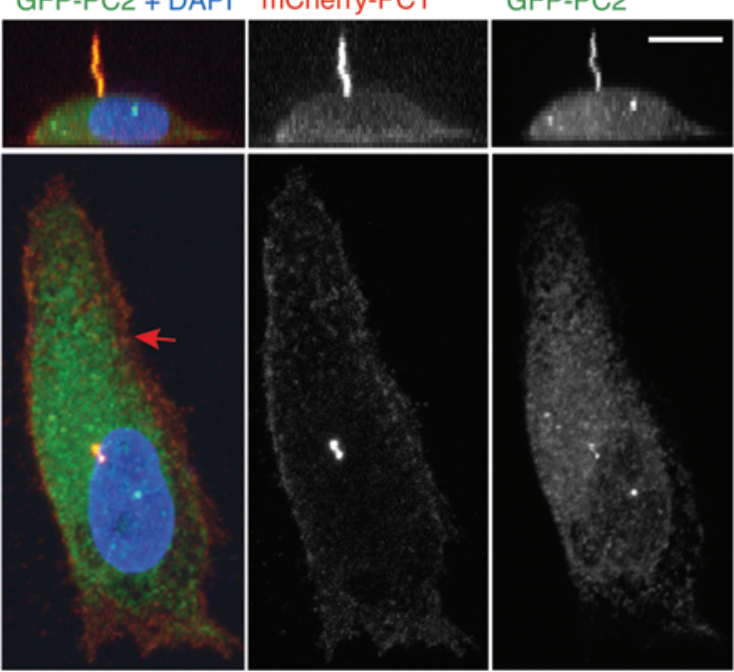

Surface

mCherry-PC1

GFP-PC2 + DAPI

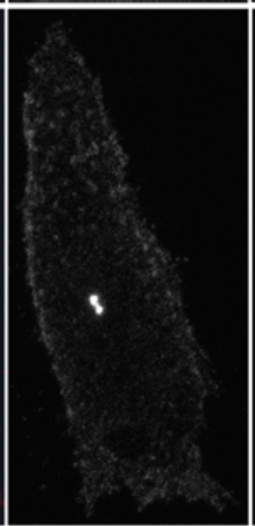

Surface mCherry-PC1
D

\section{Surface}

mCherry-PC1

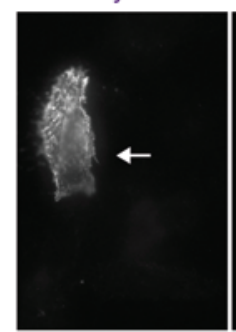

GFP-PC2

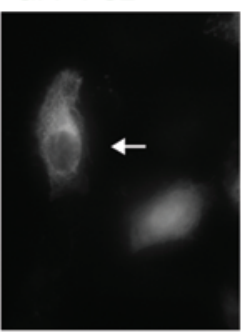

mCherry-PC1
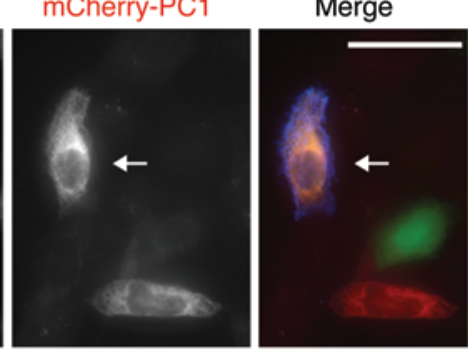

E

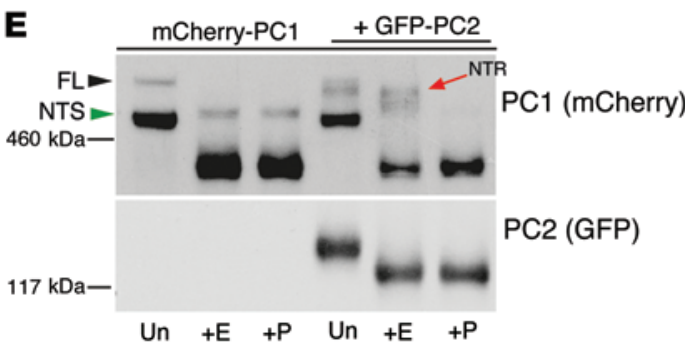

Figure 3. PM and cilia colocalization of PC1 and PC2. (A) Diagram of mCherry-PC1 and GFP-PC2 fusion proteins used in the IF experiments. (B) Confocal images of mCherry-PC1- and GFP-PC2-cotransfected RCTE cells showing prefixation surface labeling of PC1 (mCherry antibody) in live cells or all PC1 after permeabilization (total mCherry), with PC2 (GFP) and DAPI. Scale bar: $10 \mu \mathrm{m}$. Peripheral overlapping PC1/PC2 punctae are indicated with yellow arrows, with colocalization also seen in the ER. (C) Optical sectioning (z-stack, XZ plane) of confocal image of ciliated RCTE cells cotransfected with mCherry-PC1 and GFP-PC2 and subjected to prefixation PC1 labeling (mCherry antibodies). Surface PC1 and PC2 colocalized in primary cilia, while PC1 signal was also seen on the PM (red arrow). Scale bar: $10 \mu \mathrm{m}$. (D) Low-magnification image of surface-labeled RCTE cells cotransfected with mCherry-PC1 and GFP-PC2, showing mCherry-PC1 detected on the surface only in cells also expressing GFP-PC2 (arrows). Scale bar: $50 \mu \mathrm{m}$. (E) Deglycosylation analysis of RCTE cells expressing mCherry-PC1 alone or cotransfected with GFP-PC2. Mature mCherry-PC1 (PC1-NTR) was detected only in cells cotransfected with GFP-PC2, while cleaved ER-resident mCherry-PC1 (NTS) accumulated in the absence of PC2. Representative blots are shown from 3 independent experiments.

a corresponding increase in PC1-NTS. Consistent with $P k d 1^{R C}$ being partially a GPS/GAIN cleavage mutant, more PC1-FL was also seen with this model (ref. 20 and Figure 5, A and B). Addition of the $P k d 2^{+/-}$genotype to these animals resulted in an additional modest depletion of PC1-NTR (to $30 \%$ of WT), a $22 \%$ reduction compared with $P k d 1^{R C / R C}$ (Figure 5, A-C). However, we found it difficult to accurately measure PC1-NTR in those MEFs because of the low abundance of this $\mathrm{PC} 1$ glycoform, and the $22 \%$ reduction did not reach statistical significance from $P k d 1^{R C / R C}$ alone (Figure $5 \mathrm{C})$. To better quantify the reduction, we measured the PC1-NTR/ NTS ratio, thus providing an internal control. That analysis (Figure 5D) showed a significant difference in the PC1-NTR/NTS ratio when the $P k d 2^{+/}$allele was introduced. Taking these results together, we determined that a $50 \%$ reduction of PC2 $\left(\mathrm{Pkd}^{+/-}\right)$ resulted in a nonequimolar reduction of PC1-NTR by about $25 \%$ in the WT and $P k d 1^{R C / R C}$ setting. 

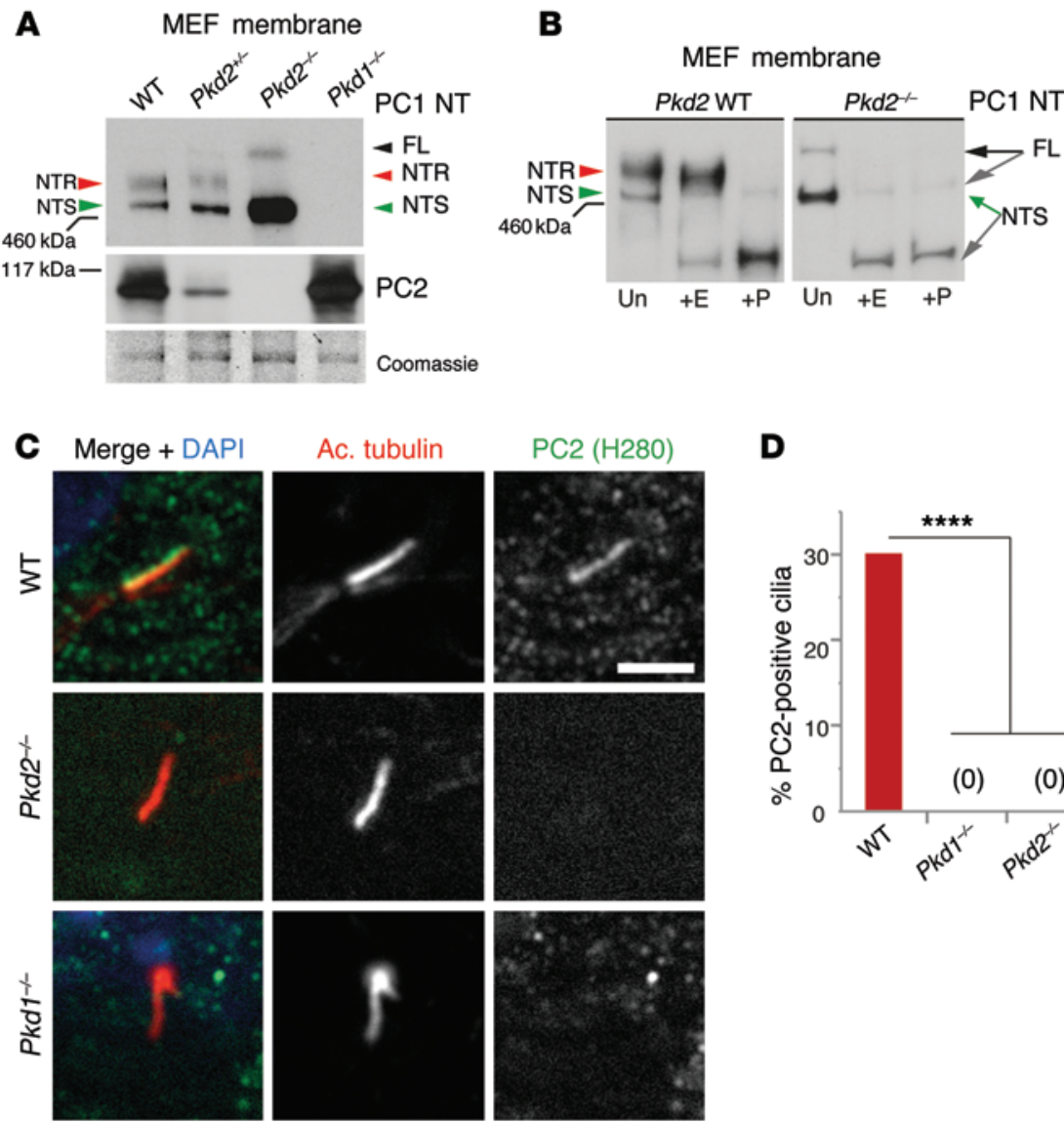

Figure 4. PC1 maturation and trafficking depend on PC2. (A) IB of membrane-purified proteins from MEFs derived from WT, $P k d 2^{+/-}, P k d 2^{-/-}$, and $P k d 1^{-/-}$ embryos detected with PC1 NT or PC2 antibodies. A Coomassie-stained loading control is shown. PC1NTR was completely absent and PC1-NTS elevated in $\mathrm{Pkd2}^{-{ }^{--}}$cells. Representative blots are shown from 3 independent experiments. (B) Clycosylation analysis of WT and $\mathrm{Pkd2}^{-/-}$MEFs showing that PC1NTR was absent and PC1-NTS elevated in $\mathrm{Pkd2}^{-1-}$ cells compared with WT MEFs. Representative blots are shown from 3 independent experiments. (C) IF detection of cilia (acetylated $\alpha$-tubulin, Ac. tubulin) and PC2 (H280) in WT, $P k d 2^{-1-}$, and $P k d 1^{-/-}$ MEFs (scale bar: $10 \mu \mathrm{m}$ ). (D) Quantification of these localizations ( $n=50$ cilia). PC2 was found on $30 \%$ of WT cilia but not on $P k d 2^{-/-}$or $P k d 1^{-/-}$cilia, indicating a crucial role for PC1 in PC2 cilia localization in MEFs ( ${ }^{* * *} P=0.0001$ by 2 -tailed Fisher's exact test).
Reduced PC2 dosage worsened PC1-dependent PKD severity. Previous studies of $\mathrm{Pkd1}^{+/-} \mathrm{Pkd2^{+/ }}$ mice showed that they developed very mild cystic disease (with single heterozygotes developing practically no cysts) (46). In this bilineal combination we estimated a PC1-NTR level of about $40 \%$ of WT (Figure 5A). Analysis of the $P k d 1^{R C / R C}$ model showed the presence of only the mutant PC1 protein, and reduction of PC1-NTR by about $65 \%$ resulted in more robust cyst development (20). We therefore analyzed the consequences of addition of the $P k d 2^{+/-}$allele to the $P k d 1^{R C / R C}$ background (PC1-NTR reduction to $30 \%$ of WT; Figure $5 \mathrm{C}$ ) for the cystic phenotype in 4-month-old C57BL/6 inbred animals (C57BL/6 inbred $P k d 1^{R C / R C}$ mice have a milder phenotype than do mice in the outbred state [ref. 57]). Percentage of kidney weight/ body weight (\% KW/BW) and cystic index (percentage cystic area) showed a significant increase in renal disease severity in the bilineal mice (Figure 6, A-C, and Supplemental Table 1), although they were much less severe than in the $P k d 1^{R C /-}$ model, where PC1-NTR is estimated to be reduced to about $20 \%$ of WT (20). Neither blood urea nitrogen nor serum creatinine was significantly elevated in these animals with relatively early-stage disease (Figure 6D and Supplemental Table 1). To further test whether the level of PC2 influenced disease severity, the $P k d 1^{R C / R C}$ model was bred with mice with the hypermutable $P k d 2^{\text {WS25 }}$ allele (58), further lowering PC2 levels in the total kidney and likely removing all PC2 in some cysts in the $P k d 2^{W S 25 /-}$ model. The $P k d 1^{R C / R C} P k d 2^{W S 25 /+}$ model was, as expected, intermediate between the $P k d 1^{R C / R C}$ and $P k d 1^{R C / R C}$ $P k d 2^{+/-}$genotypes, while the $P k d 1^{R C / R C} P k d 2^{W S 25 /-}$ combination resulted in rapidly progressive disease, significantly more severe than all other genotypes (Figure 6, A-D, and Supplemental Table 1). These results suggest that below a PC1-NTR threshold, reduction in the level of PC2 by $P k d 2$ mutation results in more severe disease and the severity is correlated with the level of PC1-NTR.

Defining the C-tail region that is critical for PC1 maturation. Previous studies found that the PC1-PC2 interaction depends on the cytoplasmic CT of PC1 (31). To test whether the PC1-CT is required for GPS/GAIN cleavage (17) and maturation of PC1, we analyzed glycosylation of endogenous PC1 truncation mutants. MEFs from a lethal $P k d 1$ knockout with a frameshift at exon $31\left(P k d 1^{d e l 31 / d e l 31}\right)$, truncating PC1 in the 2nd cytoplasmic loop, revealed a normal PC1-NTS product, indicating that this truncated product is folded, inserted into the membrane, and GPS/GAIN cleaved, although a truncated, GPS/GAIN-noncleaved product (tFL) is evident (Figure 7, A and B). However, no mature, PC1-NTR product was detected (Figure 7B). IP with PC2 confirmed that the truncated PC1 did not interact with PC2 (Figure 7C). Two additional truncating mutations in the extracellular part of PC1, the heterozygous null mouse model $P k d 1^{\text {dell7/+ }}$ (WT/del17; Figure 7, D and E) (59) and a translocation in exon 15 in PKD1 patient 77-2 (WT/tr15; Figure 7, F and G) (60), resulted in the truncated PC1-NT (tNT) product remaining EndoH sensitive, with no mature product detected.

Given these results and previous studies $(31,32)$, we tested whether mutants including or excluding the PC1-PC2 interaction domain (PC1-CC) matured. PC2 was cotransfected with PC1 constructs containing nonsense mutations before (p.E4211X), 
A

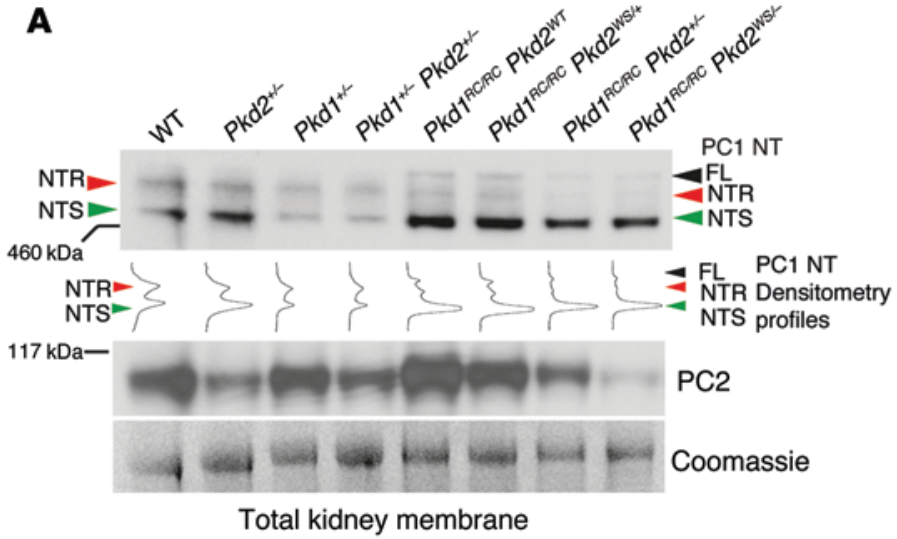

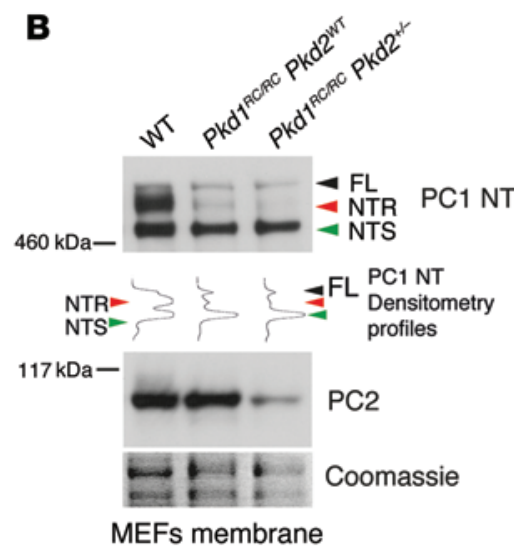
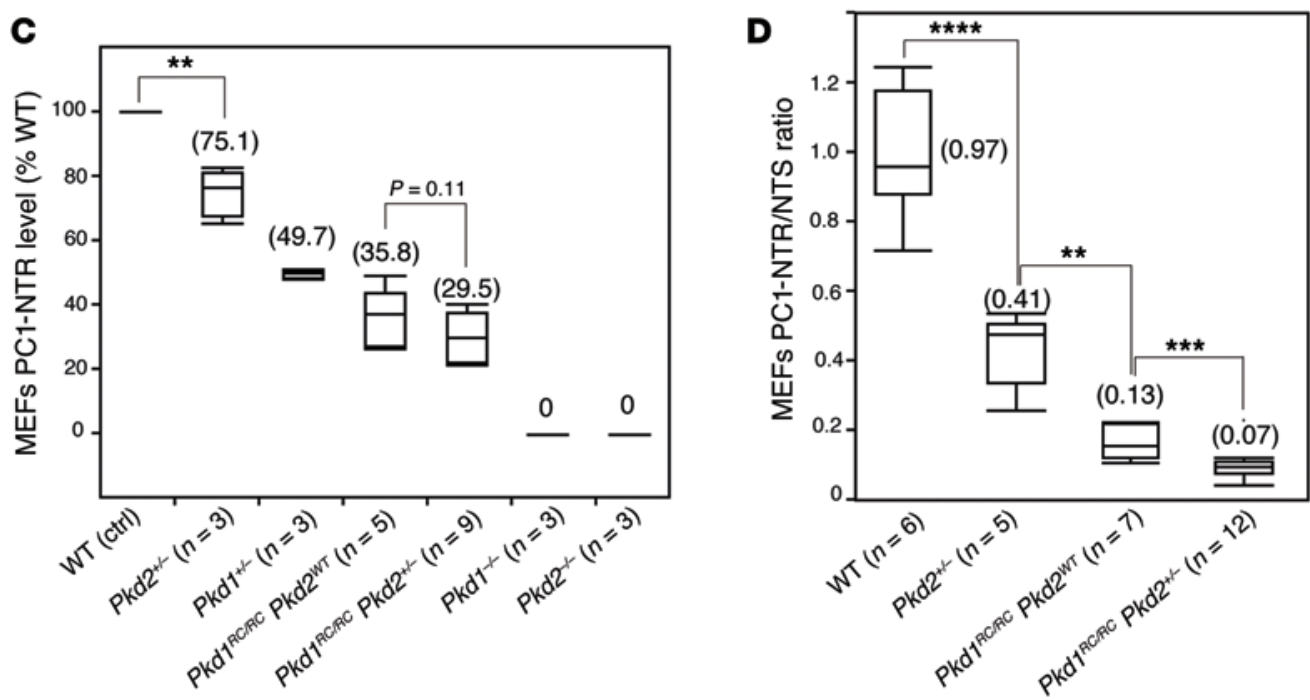

Figure 5. Maturation of PC1 is associated with the dosage of PC2. (A) Membrane protein purified from P9 mouse kidneys of WT, $P k d 2^{+/-}, P k d 1^{+/-}$, and bigenic combinations with the $P k d 7^{R C / R C}$ genotype and $P k d 2^{\text {WS25 }}$ allele assayed by SDS-PAGE and probed with PC1 NT and PC2 antibodies. Densitometric profiles of the NT products are shown with a Coomassie-stained loading control. Reduction of $P k d 2$ reduced the level of PC1-NTR, while $P k d 1$ reduction lowered the level of both products. Note in $P k d 1^{R C / R C}$ animals that the PC1-FL product is more evident, consistent with the previously described partial cleavage defect (20). Representative blots are shown from 3 independent experiments. (B) IB of membrane-purified protein from WT, $P k d I^{R C / R C} P k d 2^{+/+}$, and $P k d 1^{R C / R C} P k d 2^{+/-}$ MEFs detected with PC1 NT or PC2 antibodies showing the PC1-FL, PC1-NTR, and PC1-NTS glycoforms, PC2, and control Coomassie band. Representative blots are shown from 3 independent experiments. (C) Quantification of PC1-NTR from MEFs with various Pkd1 and Pkd2 genotypes. Results were derived from a minimum of 3 independent IBs and biological replicates obtained from 2 separate crosses (numbers $[n]$ indicated) and compared with the WT average from each group, with significance determined by the Student's $t$ test. (D) Relative ratio of PC1-NTR to NTS expression for indicated MEF genotypes (numbers $[n]$ indicated). The significance of the difference between means was compared using the Student's $t$ test. For $\mathbf{C}$ and $\mathbf{D}$, quartile box plots represent the median, quartiles, and minimum/maximum range, with means of each group in parentheses. ${ }^{* *} P<0.01$; ${ }^{* * *} P<0.001$; ${ }^{* * * *} P<0.0001$.

within (p.E4220X, p.Q4247X), or after (p.G4257X) the coiled-coil domain. Analysis with deglycosylation showed a clear PC1-NTR product if the deletion occurred after or at the end of the coiledcoil domain and no product if the coiled-coil domain was completely deleted (Supplemental Figure 5, A and B). These data suggest that loss of the $\mathrm{PC} 1$ coiled-coil domain, the site of complexing with PC2, regulates PC1 maturation.

Deletion of CC2 and missense mutations in PC2 influence PC1 maturation. To obtain more specific information about how PC2 determined the maturation of PC1, the glycosylation pattern of exogenous PC1 was analyzed when variously mutated forms of PC2 were coexpressed. As expected, loss of the entire C-tail (CT; PC2-p.L703X) completely prevented PC1 maturation, but interestingly, deletion of the proximal coiled coil and the $\mathrm{Ca}^{2+}$ bind- ing domain of the $\mathrm{EF}$ hand (delEF+CC1), previously found to be important for PC2 oligomerization and function (34), had no effect on PC1 maturation (Figure 8, A-D, and Supplemental Figure 6). In contrast, truncation of PC2 after the ER-retention motif and before CC2 (p.S835X), or in-frame deletion of the CC2 (delCC2), disrupted PC1 maturation, consistent with the suggested role of CC2 in complexing with PC1 (34). Analysis of PC2 on a nondenaturing gel showed monomers when CC2 was deleted (but not CC1), which were not seen in the WT, supporting previous findings that $\mathrm{CC} 2$ but not $\mathrm{CC} 1$ promotes PC2 dimerization (ref. 34 and Supplemental Figure 7). These results indicate that PC2-CC2 is necessary for efficient PC1 maturation.

Although most PKD2 patients harbor truncating mutations, about $10 \%$ have missense changes (61). To determine whether 
A

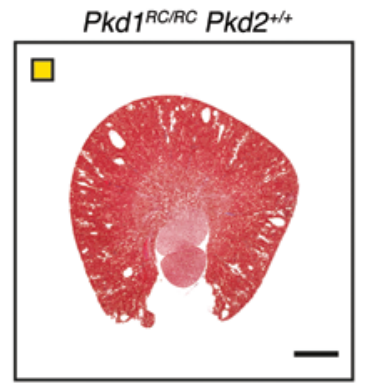

Pkd1 ${ }^{\text {RC/RC }}$ Pkd2 ${ }^{\text {Ws25/ }}$

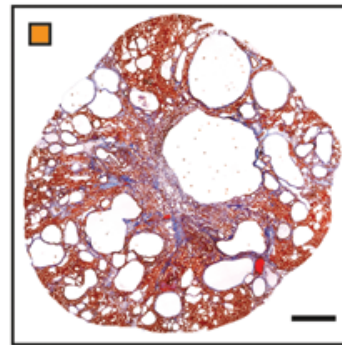

B

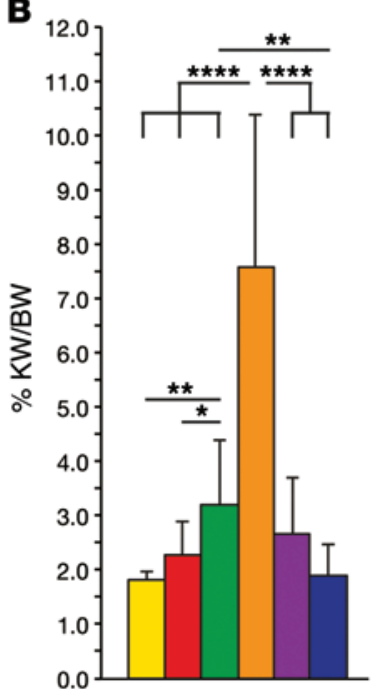

Pkd1 ${ }^{\text {RC/RC }}$ Pkd2WS25/*

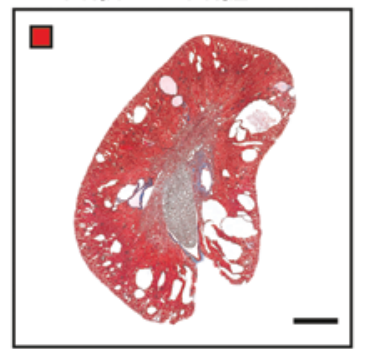

$P k d 1^{\mathrm{RC} / \mathrm{P}} \mathrm{Pkd} 2^{\mathrm{WS} 25 /-}$

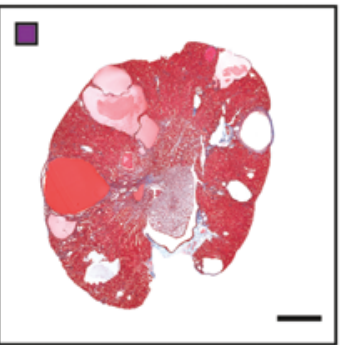

C

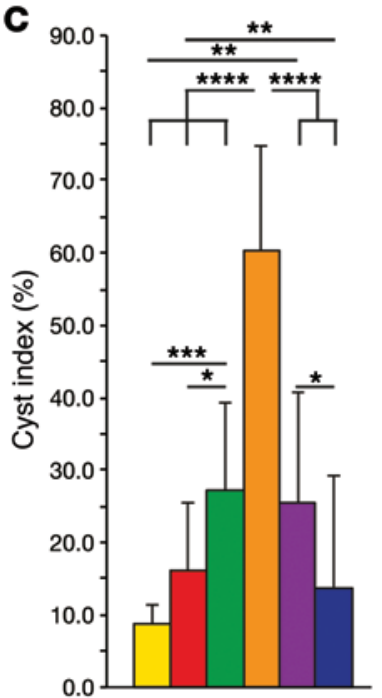

Pkd1 ${ }^{R C / R C} P k d 2^{+/}$

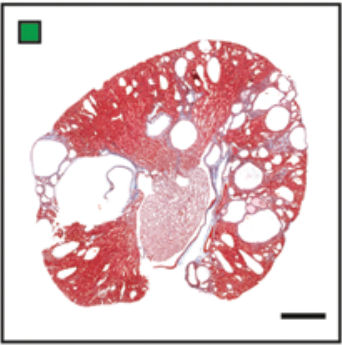

$\mathrm{Pkd1}^{+/+} \mathrm{Pkd2}{ }^{\text {Ws25/- }}$

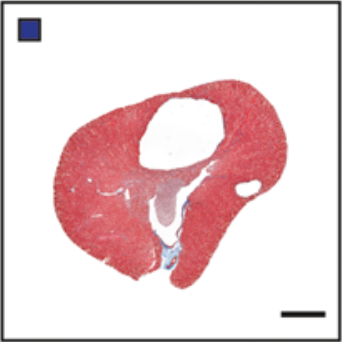

D

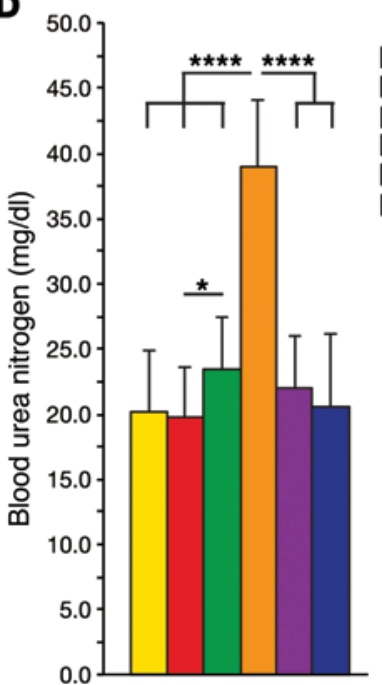

$\square P k d 1^{\text {ACACC }} P k d 2^{* / *}(n=6 \mathrm{~F}, 6 \mathrm{M})$

Pkd1 ${ }^{\text {AC/AC }} P k d 2^{W S 25 / 4}(n=6 \mathrm{~F}, 6 \mathrm{M})$

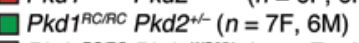

Pkd1 ${ }^{\text {ACACAC }} P k d 2^{\text {Ws25/- }}\left(n=4 \mathrm{~F}, 6 \mathrm{M}^{+}\right)$

$P k d 1^{1{ }^{R / 4}}$ Pkd2 $^{\text {WS25/- }}(n=6 \mathrm{~F}, 6 \mathrm{M})$

Pkd1 $^{+/+}$Pkd2 $^{\text {WS25/- }}(n=5 \mathrm{~F}, 7 \mathrm{M})$

Figure 6. Pkd2 depletion aggravates the Pkd1RC/RC cystic phenotype. (A) Masson trichrome-stained kidney cross sections of 4-month-old mice of the $P k d 7^{R C / R C}$ genotype with the addition of $P k d 2^{\text {WS25/+}}, P k d 2^{+/-}$, or $P k d 2^{\text {WS25/- }}$, and $P k d 2^{\text {WS25/- }}$ mice with the $P k d 7^{R C /+}$ genotype. PKD severity and fibrosis worsened in bigenic mice, with evidently more severe disease in the $P k d 1^{R C / R C} P k d 2^{+/-}$genotype, corresponding to PC1-NTR levels of about 30\% (Figure 5C). However, the $P k d 7^{R C / R C} P k d 2^{W S 25 /-}$ combination resulted in the most severe disease. Scale bar: $1 \mathrm{~mm}$. (B-D) Graphical representations of \%KW/BW (B), cystic index (C), and blood urea nitrogen (D) of the various genotypes quantify the increased disease severity with Pkd1/Pkd2 combined phenotypes (see Supplemental Table 1 for details). Error bars depict \pm SD. ${ }^{*} P<0.05,{ }^{* *} P<0.01$, ${ }^{* *} P<0.001,{ }^{* * *} P<0.0001$ using a 2-way ANOVA with Student's $t$ test. F, female; M, male. ${ }^{+}$Note that 5 of 10 Pkd1 ${ }^{R C / R C} P k d 2^{\text {WS25/- }}$ animals died before 4 months (F: P42, P74; M: P38, P51, P79).

missense PC2 mutations alter PC1 maturation, we generated 4 of the most common or best-characterized substitutions: p.R322Q, p.R322W, p.W414G, and p.D511V (ADPKD Mutation Database: PKDB; http://pkdb.mayo.edu). Interestingly, the data differed between mutations, with some, like p.W414G, completely eliminating PC1 maturation, while the apparent channel-dead mutant p.D511V (6) still facilitated some PC1-NTR generation; none seemed to effect PC2 oligomerization (Figure 8, C and D, and Supplemental Figure 6). IP of doubly transfected cells with PC2 (GFP) showed that co-IP with PC1 was greatly reduced if CC2 was deleted, but PC2 with missense mutations still complexed with PC1 (Supplemental Figure 7).

\section{Discussion}

Here we show that the maturation of PC1 resulting in the development of the critical PC1-NTR product is regulated by PC2 and, hence, that PC2 plays a central chaperone-like role in PC1 processing. The strengths of the study are that we have largely analyzed endogenous PC1 and PC2 in cells, tissue, and whole animals in the WT setting and in the context of specific mutations that remove, reduce, or alter one or both ADPKD proteins. The importance of interplay between PC1 and PC2 manifests as significantly more severe disease in bigenic models, emphasizing the importance of the interaction to understanding the cystogenic process. 
A

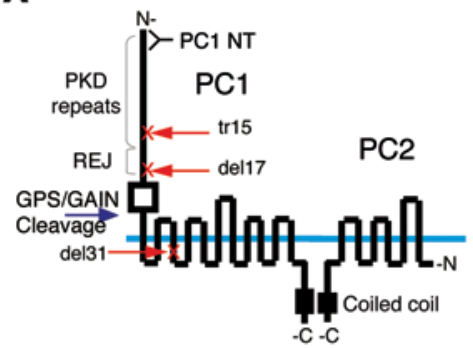

B

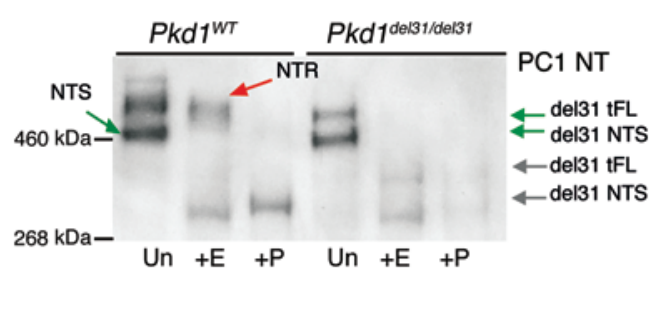

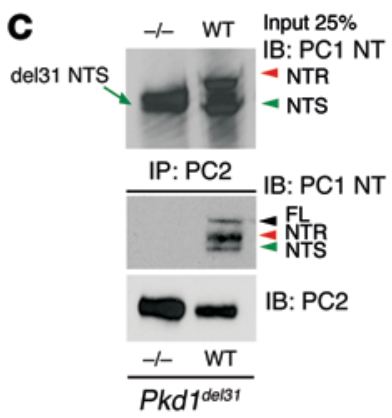

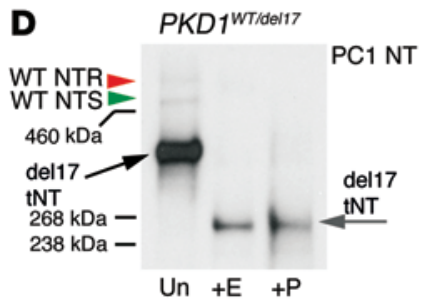

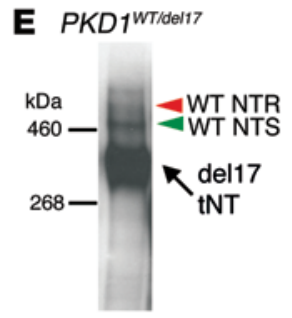

PC1 NT long exp.

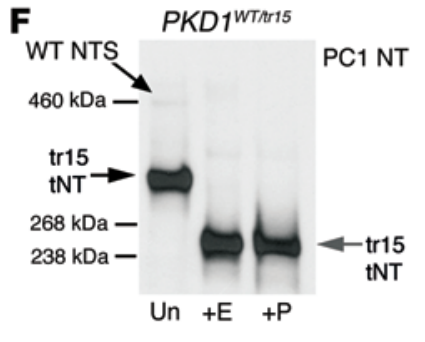

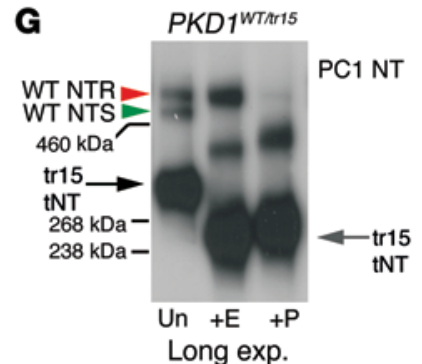

Figure 7. Analysis of maturation of endogenous PC1 truncation mutants. (A) Diagram showing approximate locations of the truncations in PC1 and the locations of the REJ domain, PKD repeats, and CT-CC. (B) Glycosylation analysis of MEFs isolated from WT and Pkd1/el37/del31 embryos detected with the PC1 NT antibody. The del31 mutation truncated PC1 after the GPS/GAIN cleavage site, and since the truncated protein was cleaved, an NT as well as a truncated FL (tFL) product were observed. EndoH analysis shows that PC1-NTS, but not PC1-NTR, was generated. Representative blots are shown from 3 independent experiments. (C) IP of endogenous PC2 (H280) in WT and Pkd 1del31/del31 (-/-) MEFs shows that PC2 did not coimmunoprecipitate with the del31 mutant PC1. Lysate control is shown above. Representative blots are shown from 3 independent experiments. (D-C) Pkd $7^{\text {dell7/+ }}$ (WT/del17) adult mouse kidney ( $\mathbf{D}$ and $\mathbf{E})$ and human fibroblasts from a female ADPKD patient with an extracellular truncation due to a translocation in exon 15 (WT/tr15; 77-2) (F and G), disrupting PC1 extracellularly in the REJ domain or PKD1 repeats, respectively. (D, F, and G) Glycosylation analysis comparing untreated (Un), EndoHdigested (+E), and PNGase F-digested (+P) protein. In all cases, only the EndoH-sensitive truncated product (PC1-tNTS) was seen, with no PC1-tNTR glycoform. In del17 and tr15, the PC1 truncated product was expressed at a much higher level than that detected WT, and so longer exposures are shown (E and $\mathbf{G}$ ) to visualize the WT allele/products. Representative blots are shown from 3 independent experiments.

Previous analysis of the hypomorphic Pkd1: p.R3277C allele showed that the pathomechanism was related to a lower level of PC1-NTR, due to reduced GPS/GAIN cleavage and defective protein folding (20). Here we show that PC1 truncation within the transmembrane region results in a stable mutant product, integrated into the membrane and cleaved at the GPS/GAIN domain. Truncation before the first transmembrane region resulted in a stable, sometimes greatly overexpressed, protein, which in the case of the tr15 mutant product is overexpressed at the mRNA level (60). Overexpressed truncated protein may be due to loss of the PEST sequence in the PC1-CT (32), although not all truncated PC1 proteins are overexpressed (del31). None of the truncated proteins generate mature $\mathrm{PC} 1$ products, likely because of their inability to interact with PC2; therefore, we predict that PC1 truncation mutants preceding the coiled-coil domain do not mature and fail to reach the cell surface.

Our data indicate that PC1 and PC2 initially interact in the ER before GPS/GAIN cleavage and that this interaction is central to the subsequent maturation of PC1, including exiting from the ER and cell surface localization. In contrast to previous studies (17), efficient GPS/GAIN cleavage occurred when no PC2 was present, and truncated PC1 was still efficiently cleaved even without the ability to interact with PC2. We corroborate that PC2 influences cell surface localization of PC1 (17), and show an absolute requirement of that interaction for PC1 surface localization. Some, but not all, PC2 pathogenic missense mutations failed to facilitate PC1 maturation, even though the missense mutants were able to interact with PC1 and form PC2 oligomers. Hence, further analysis is required to fully understand their pathomechanism.

Our studies provide insight into the subcellular localizations of specific PC1 and PC2 glycoforms. All detectable PC2 appeared to be EndoH sensitive (even after enrichment by PC1 IP) and mostly ER resident (13). However, we describe a corresponding ER-resident form of PC1 (NTS) that complexes with PC2. This may be an intermediate form associated with the maturation of PC1, but suggest an ER-localized form of the polycystin complex. Our studies show that PC1-NTR was secreted in total media, consistent with the PC1 secreted on ELVs in urine and from cultured cells being PC1-NTR $(14,20)$. We also found that secretion of PC1 was PC2 dependent. We confirmed that exogenously expressed PC1 and PC2 $(26,27)$, and endogenous PC2, localize to cilia and showed that PC1-NTR accumulated in a cilia enriched fraction with EndoH-sensitive PC2. This is consistent with PC2 reaching the cilium by bypassing the mid-/trans-Golgi, independent of PC1 $(19,35)$. Interestingly, our data also indicated in MEFs that the presence of PC2 on the cilium requires endogenous $\mathrm{PC} 1$, which may be consistent with reduced ciliary PC2 on PKD1 induced pluripotent stem cells (iPS) and derived cells (62). However, the literature is inconsistent in this regard $(15,17,19,35)$; different cell types may behave differently, and so further studies are required. We provide evidence from fractionation of the endogenous protein and IF of exogenous protein that a significant proportion of 

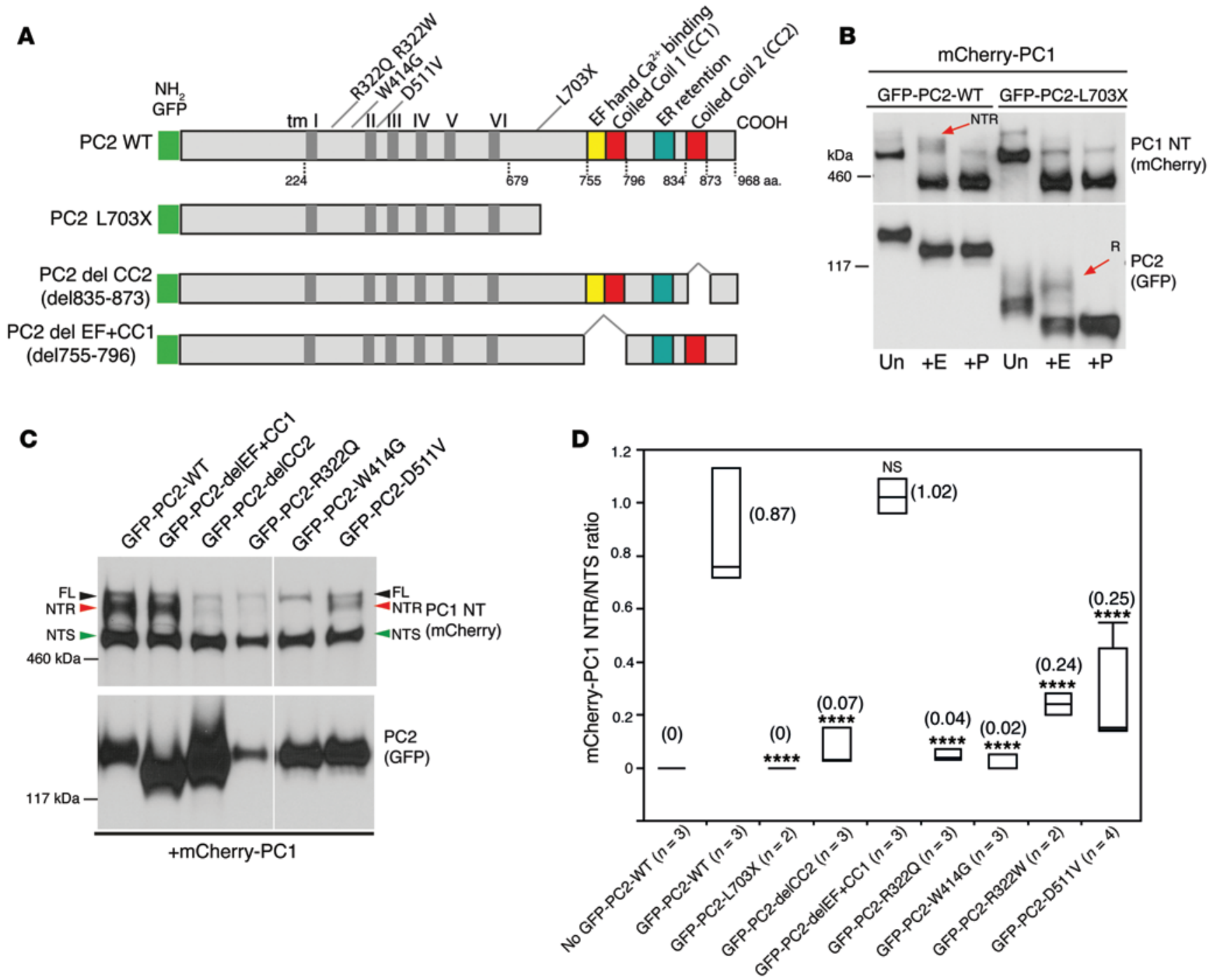

Figure 8. Deletion of CC2 and missense mutations in PC2 influence PC1 maturation. (A) Diagram of WT and mutant human PC2 used in the cotransfection experiments (B and $\mathbf{C}$ ) showing the positions of domains, deletions, and aa substitutions. (B) mCherry-PC1 cotransfected with WT GFP-PC2 or PC2 lacking the C-terminal cytoplasmic tail (GFP-PC2-L703X) into RCTE cells. Detection of PC1 NT (mCherry) revealed EndoH-resistant PC1-NTR (arrow) only in WT-PC2, but not in PC2-L703X, cotransfected cells. Detection of PC2 (GFP) shows that a portion of PC2-L703X was EndoH resistant (R; arrow). Representative blots are shown from 3 independent experiments. (C) Cotransfection of various GFP-PC2 mutants and mCherry-PC1 into RCTE cells showing the effect of PC2 mutations on the PC1 glycosylation pattern and PC2 products. Deletion of the EF hand and coiled coil 1 (delEF+CC1) did not disrupt PC1 maturation, but deletion of coiled coil 2 (delCC2) greatly reduced the level of the PC1-NTR product. Pathogenic missense substitutions in PC2, especially p.R322Q and p.W414G, also had a negative effect on PC1 maturation. Representative blots are shown from 3 independent experiments. (D) Quantification of the ratio of PC1-NTR to NTS glycoforms obtained from the cotransfection experiments with WT and mutant PC2 GFP constructs (C and Supplemental Figure 5A). Deletion mutants removing CC2 but not EF+CC1 largely disrupted PC1 maturation, while missense mutations also significantly disrupted maturation. $n=3$ for all except R322W ( $n=2)$ and D511V $(n=4)$. Quartile box plots represent the median, quartiles, and minimum/maximum range, with the mean of each group in parentheses. $P$ values are shown as compared with GFP-PC2-WT (control) with the Student's $t$ test; ${ }^{* * *} P<0.0001$.

PC1-NTR localizes to the PM (17). An additional study published after the submission of this manuscript also localized PC1-NTR to the PM (63), and suggested a PC1-NT product not tethered to PC1-CT. Our results suggest that the PC1-PC2 complex formation is required for ER exit of both proteins and imply that PC2 plays a role in folding/quality control of PC1. This is consistent with the observation that PC1 structure is altered upon PC2 binding (64).

The major conclusion from this study is that PC2 acts as an important chaperone for PC1 maturation and localization. In PKD1, the level of PC1-NTR is critical for disease severity (20), and here we show that the PC2 reduction in PKD2 results in a nonequimolar reduction of PC1-NTR, which likely modifies the PKD2 phenotype. The bigenic mouse studies show that addition of a single $P k d 2$ mutant allele (null or WS25) had a significant impact on the $P k d 1^{R C / R C}$ phenotype, with corresponding changes seen with a single $P k d 1^{R C}$ allele on the $P k d 2^{W S 25 /-}$ background. Once below a critical PC1-NTR threshold, $35 \%-40 \%$ of WT in mice (and probably $\sim 50 \%$ in humans), small additional reductions are associated with significant phenotypic changes (20). Of interest is the significantly different disease severity between $P k d 1^{+-} P k d 2^{+/-}$and $P k d 1^{R C / R C}$ 
mice. This may be because in this combination no WT $P k d 1$ is present, although the level of PC1-NTR (5\% reduced) may be most significant $(46,57)$. A combined effect of both loci is consistent with the observations of $P K D 1$ somatic mutations in $\mathrm{PKD} 2$ patient cysts (65), and vice versa (66), likely acting by lowering PC1-NTR below a cystogenic threshold and so favoring cyst development.

Overall our studies emphasize that PC1-NTR is the cell surface glycoform of PC1, that maturation of PC1-NTR requires the presence of PC2 in a dose-dependent manner, and that the renal disease severity in $P k d 1 / P k d 2$ models parallels the level of PC1-NTR.

\section{Methods}

Crude membrane protein purification. Cells were washed with PBS, scraped and reconstituted in LIS buffer $(10 \mathrm{mM}$ Tris $\mathrm{HCl}$ [pH 7.4], $2.5 \mu \mathrm{M} \mathrm{MgCl}_{2}, 1 \mathrm{mM}$ EDTA) and protease inhibitors (Complete; Roche), and allowed to swell. Cells were then homogenized by repeated passage through a 25.5-gauge needle and centrifuged at 2,200 $\mathrm{g}$ for $5 \mathrm{~min}$ utes. Supernatant was collected, centrifuged at 20,000 $g$ for $30 \mathrm{~min}$ utes, and the pellet saved as a crude membrane fraction. All procedures used prechilled containers and were performed in the cold room.

Generation of total cellular membrane (PM+ microsomes). Cells were washed with PBS, scraped and reconstituted in LIS buffer, and allowed to swell. Cells were then homogenized with a tight-fitting Dounce homogenizer and passed once through a 25.5-gauge needle. Homogenates were made iso-osmotic by addition of sucrose to $320 \mathrm{mM}$, and membranes were centrifuged at 10,000 $g$ for 15 minutes and the supernatants saved.

Sucrose purification of membrane protein. Purified membrane protein/microsomes were overlaid on a sucrose cushion (35\%) and centrifuged at 100,000 $g$ for 2 hours. Membrane protein was collected from the sucrose interface.

Gradient fractionation of renal epithelial cells. RCTE cells were washed in ice-cold PBS, scraped, collected by centrifugation, reconstituted in LIS buffer, and homogenized with a tight-fitting Dounce homogenizer. Lysates were made iso-osmotic by addition of sucrose/ LIS solution to $250 \mathrm{mM}$ and cleared of whole cells/nuclei by centrifugation at 10,000 $\mathrm{g}$ for 10 minutes. Cleared supernatants were overlaid on $2 \%$ to $30 \%$ OptiPrep gradients and subjected to ultracentrifugation at 48,000 $g$ for 18 hours. Gradients were prepared using the gradient-maker function of the Gradient Master (BioCorp). Fractions of $500 \mu \mathrm{l}$ were collected from the top using the fractionation function of the Gradient Master.

Purification of media-derived protein. MEFs were grown to $100 \%$ confluence in $15-\mathrm{cm}$ plates and washed 3 times in PBS, and medium was replaced with protein-free chemically defined (CD Hybrydoma; Invitrogen) medium supplemented with $1 \%$ clarified FBS. FBS was clarified by ultracentrifugation for 2 hours at 100,000 $\mathrm{g}$ and passed through a 50,000-Da-cutoff centrifugal filter (Millipore). After 48 hours, CD medium was collected from cultured MEFs and cleared of cells and membranes by successive centrifugations at 1,000 and $10,000 \mathrm{~g}$ for 5 and 15 minutes, respectively. Supernatants were then concentrated using 50,000-Da-cutoff centrifugal filters (Millipore) (Supplemental Figure 4A).

Cloning mCherry-PC1 and GFP-PC2 constructs. pTagGFP2::PKD2 was constructed in the pTagGFP2 vector (Evrogen). A long linker SGGGGTGGGSGG was cloned in frame after the vector BspEI site and a human PC2 cDNA (NM_000297.3) inserted seamlessly after the linker. To generate PKD2, c.2107_2109CTT>TAA (p.L703X), c.2503_2505TCT>TAA (p.S835X), c.2263_2388del (p.755_796del, delEF+CC1), c.2503_2619del (p.835_873del, delCC2), c.1240T>G (p.W414G), c.964C>T (p.R322W), c.965G>A (p.R322Q), c.1532A>T (p.D511V) mutagenesis was performed using Q5 polymerase (NEB). mCherry::PC1 was generated by insertion of an mCherry-RFP domain after (C-terminal to) the native PC1 signal peptide but $\mathrm{N}$-terminal to the leucine-rich repeat-associated cysteine-rich cluster. Therefore, the mCherry protein was expressed on the extreme N-terminal tip of the mature PC1 molecule. To generate PKD1, c.12631G > T (p.E4211X), c.12658G > T (p.E4220X), c.12736C >T (p.Q4247X), c.12788G>T (p.G4257X) mutagenesis was performed using Q5 polymerase.

Semiquantitative Western blotting. Membrane protein from crude purification was quantified (BCA Protein Kit; Thermo Scientific) and samples diluted to $2 \mu \mathrm{g} / \mu \mathrm{l}$ before Western blotting. Equal concentrations of membrane protein $(25 \mu \mathrm{g})$ were subjected to SDS-PAGE in $3 \%-8 \%$ Tris-acetate gradient gels (Invitrogen) for 2.5 hours at $150 \mathrm{~V}$. Gels were washed and transferred onto PVDF membranes using a wet transfer box (Bio-Rad) for at least 4 hours at a constant voltage of $80 \mathrm{~V}$. Transfer efficiency and equal loading were confirmed with Coomassie blue (Bio-Safe; Bio-Rad) staining of gels. PVDF membrane blots were blocked in 5\% nonfat milk in Tris-buffered saline plus Tween (TBST) and exposed to primary antibodies overnight at $4^{\circ} \mathrm{C}$. For quantification, each experiment was compared with a WT control using densitometry (ImageJ) and normalized to total membrane protein obtained from Coomassie-stained gels and/or SYPRO Ruby-stained PVDF membrane.

Nondenaturing Western blotting. Nondenaturing gel electrophoresis was performed using NativePAGE 3\%-12\% Bis-Tris system (Invitrogen) according to the manufacturer's instructions.

Glycosylation analyses. Purified membrane protein and IP eluates were deglycosylated with EndoH and PNGase F kits according to the manufacturer's instructions (NEB).

Surface glycoprotein labeling and IP. Surface glycoprotein labeling of living cells was performed as described previously (67). Briefly, monolayer cells in $15-\mathrm{cm}$ plates were washed twice with ice-cold Dulbecco's PBS (DPBS), then oxidized at $4^{\circ} \mathrm{C}$ in $1 \mathrm{mM} \mathrm{NaIO}_{4}$ containing DPBS ( $\mathrm{pH}$ 7) for 30 minutes. The oxidation reaction was quenched with ice-cold $1 \mathrm{mM}$ glycerol in DPBS for 5 minutes, after which the cells were washed twice with ice-cold DPBS. Oxime ligation was performed in the presence of $10 \mathrm{mM}$ Aniline (Sigma-Aldrich) and $100 \mu \mathrm{M}$ Alkoxyamine- $\mathrm{PEG}_{4}$-Biotin (Thermo) in 1\% FBS-supplemented icecold PBS ( $\mathrm{pH}$ 7.5) buffer for 1 hour. Biotinylated cells were then washed 3 times in PBS and scraped. Reaction specificity was ensured using streptavidin-488 fluorophore staining of a small sample of cells.

Scraped cells were collected by centrifugation, subjected to crude membrane protein purification, and solubilized in IP buffer $(20 \mathrm{mM}$ HEPES [pH 7.5], $137 \mathrm{mM} \mathrm{NaCl}, 1 \% \mathrm{NP}-40,10 \%$ [wt/vol] glycerol, $2 \mathrm{mM}$ EDTA, $2.5 \mathrm{mM} \mathrm{MgCl}_{2}$ ) supplemented with protease inhibitors (Roche) for 15 minutes. Nonsolubilized protein was removed by centrifugation and discarded. Neutravidin agarose was washed in IP buffer 3 times, and $50 \mu$ l of packed agarose was added to solubilized membrane protein and agitated at $4^{\circ} \mathrm{C}$ for 2 hours. Samples were washed 3 times in IP buffer and once in ice-cold $\mathrm{H}_{2} \mathrm{O}$. Protein was eluted in either lithium dodecyl sulfate (LDS) plus Tris(2-carboxyethyl)phosphine (TCEP) or agarose subjected to deglycosylation analyses.

Immunoprecipitation with PC1 and PC2 antibodies. Crude membrane protein $(75 \mu \mathrm{g})$ was isolated as described and the resulting membrane 
pellet solubilized in IP buffer or high-salt $(500 \mathrm{mM} \mathrm{NaCl})$ IP buffer. Samples were precleared with blank A/G agarose for 2 hours. Antibodies to PC1 CT (goat anti-PKD1, Everest Biotech; rabbit BD3, Genzyme) and PC2 (H280 or YCE2; Santa Cruz Biotechnology Inc.) were added overnight. Protein/antibody mixture was then incubated with $50 \mu \mathrm{l}$ of packed washed A/G agarose (Thermo Scientific) for 2 hours. The agarose was washed 3 times in IP buffer or high-salt $(500 \mathrm{mM} \mathrm{NaCl})$ IP buffer and once in ice-cold $\mathrm{H}_{2} \mathrm{O}$, and the protein eluted in either LDS plus TCEP or agarose was split into 3 equal parts (untreated, EndoH, and PNGase F) and subjected to deglycosylation analyses. Twenty-five micrograms of input and $100 \%$ of the IP were loaded on SDS-PAGE.

Transfection of renal epithelial cells. RCTE (68) and MDCK (ATCC) cells were split 1:2 the day before electroporation and transfected at approximately $80 \%$ confluence. Transfection was performed using the A-020 (RCTE) and A-023 (MDCK) programs of the Amaxa Nucleofector II (Lonza) following the manufacturer's directions. Electroporation buffer (20 mM HEPES, $135 \mathrm{mM} \mathrm{KCl,} 2 \mathrm{mM} \mathrm{MgCl}_{2}, 0.5 \%$ Ficoll 400, pH 7.6) was supplemented with 1:100 volume $200 \mathrm{mM} \mathrm{ATP} / 500$ $\mathrm{mM}$ glutathione immediately after electroporation. Cells were then recovered at $33^{\circ} \mathrm{C}$ overnight to promote folding and expression of the large, mCherry-PC1, protein, then washed and returned to $37^{\circ} \mathrm{C}$. Stable mCherry-PC1 and GFP-PC2 expression was achieved by growing cells for 7 days after transfection in cell culture media supplemented with G418 (400 $\mu \mathrm{g} / \mathrm{ml})$.

Confocal microscopy, immunofluorescence, and surface PC1 labeling. RCTE cells were grown on glass cover slips, washed once with DPBS, fixed in 3.5\% paraformaldehyde (PF) for 30 minutes, and permeabilized with $0.1 \%$ Triton in DPBS ( $\mathrm{pH}$ 7.5). Cells were washed again in PBS and incubated in blocking buffer (10\% normal goat serum, 1\% BSA, 0.1\% Tween in PBS [pH 7.5]) for 30 minutes. After 3 PBS washes, primary antibodies were added in IF buffer (1\% BSA, PBS [pH 7.5], $0.1 \%$ Tween) for 2 hours at room temperature or overnight at $4^{\circ} \mathrm{C}$ with gentle agitation. After 3 PBS washes, conjugated secondary antibodies (Alexa Fluor; Invitrogen) were added for 1 hour. DAPI was added for 1 minute to stain cell nuclei.

Surface labeling of mCherry-PC1 was performed as follows. Transfected RCTE cells were cooled at $4^{\circ} \mathrm{C}$ for 15 minutes and washed once in ice-cold PBS, and prechilled mCherry antibody (BioVision), diluted in $0.5 \%$ BSA in PBS, was added for 30 minutes at $4^{\circ} \mathrm{C}$. Cells were then fixed in $3.5 \% \mathrm{PF}$ and conjugated secondary antibody added for 30 minutes in IF buffer. Confocal microscopy was performed using a Zeiss Axiovert equipped with Apotome. High-quality confocal imaging was performed using a Zeiss laser-scanning (LSM780) microscope.

pH-shift/SDS immunofluorescence. The fixation/antigen retrieval method of $\mathrm{pH}$-shift/SDS immunofluorescence was performed to visualize endogenous PC2 in MEFs (Figure 4C). Cells were grown to $100 \%$ confluence and serum starved for 48 hours, fixed in 3\% PF (pH 7.5) for 15 minutes and 4\% PF (pH 11) in $100 \mathrm{mM}$ borate buffer for 15 minutes, and then permeabilized in 1\% SDS for 5 minutes, to partially denature the protein. Subsequent antibody staining was performed as described above with the PC2 antibody (H280) staining performed overnight at a 1:200 dilution at $4^{\circ} \mathrm{C}$. Antibody specificity was ensured by comparison between WT and Pkd2-null MEFs, in which no staining was observed.

Mouse genetics and phenotype studies. The following mouse lines were used: $P k d 1$ del2 $\left(P k d 1^{+-}\right)$(69) (a gift from Shigeo Horie, Teikyo University, Tokyo, Japan); $P k d 1^{\text {tml.1Pcha }}\left(P k d 1^{R C / R C}\right)(20), P k d 2^{\text {tm2Som }}$ $\left(P k d 2^{+/-}\right)(45)$, and $P k d 2^{\text {tmISom }}\left(P k d 2^{W S 25 /+}\right)(58)$ (gifts from Stefan Somlo,
Yale University, New Haven, CT); and Pkd1 del17 (59) (a gift from Richard Sandford, University of Cambridge, Cambridge, United Kingdom). $P k d 1$ del 31 is a newly described $P k d 1$-null model where exons 31-34 are deleted and replaced with a selection cassette, mimicking the mutation in patient OX114 (P98) (60). All lines were fully inbred into the C57BL/6 strain.

For the phenotypic analysis, the animals were sacrificed at 4 months by $\mathrm{CO}_{2}$ exposure and the BWs of the animals recorded. Subsequently, blood was collected via cardiac puncture and kidneys harvested and weighed. The left kidney was flash frozen and the right kidney fixed in $4 \%$ PF. All tissues were paraffin embedded and stained (H\&E, Masson trichrome) by the Animal Histology Core (Mayo Clinic) and examined for abnormal pathologies. Cystic indices were calculated and averaged from 3 cross sections per kidney using MetaMorph software. Blood urea nitrogen was as analyzed from plasma using pHOx Ultra (Nova Biomedical), and plasma creatinine levels were measured following the BioAssay Systems protocol.

Antibodies used. The following antibodies were used: PC1 NT IgG1, 7e12 (70) (WB 1/1,000); PC1 CT Rb, BD3 (a gift of Oxana Beskrovnaya, Genzyme, Framingham, Massachusetts, USA) (IP 1/250); PC1 CT Gt, EB08670 (Everest Biotech) (IP 1/250); PC1 CT Rb, PKS-A (71); PC2 Rb, H280 (Santa Cruz Biotechnology Inc.) (WB 1/5,000); PC2 IgG2a, YCE2 (Santa Cruz) (WB 1/2,000, IF 1/500); EGFR Rb (BD Transduction Laboratories) (WB 1/1,000); acetylated $\alpha$-tubulin, IgG2b (Invitrogen) (IF 1/5,000); anti-Tag(CGY)FP (Evrogen AB121) (1/5,000 WB); mCherry Rb (5993-100; BioVision) (Surface Labeling 1/1,000); mCherry IgG2a (Novus Biologicals NB1967521C51) (WB 1/2,000); calnexin Rb (Novus Biologicals) (1/250 IF, 1/1,000 WB); ORAI-1 Rb (H-46; Santa Cruz Biotechnology Inc.) (1/1,000 WB); Arl13b (17711-1-AP; Proteintech) (1/1,000 WB); and STIM1 IgG2a (M01; Abnova) (1/2,000 WB).

Cells. RCTE $\left(P K D 1^{+/+}\right)$and 9-12 (PKD1--) cells (provided by Mahmoud Loghman-Adham, Baxter Healthcare Corporation) were previously described (68). WT, $P k d 1^{+/-}, P k d 1^{-/}, P k d 2^{+/-}, P k d 2^{-/-}, P k d 2^{R C / R C}$, $P k d 2^{R C / R C} P k d 2^{+/-}$, and $P k d 1^{d e l 31 / d e l 31}$ MEFs were generated from E13.5 mouse embryos following standard techniques. Briefly, embryos were washed twice in PBS, decapitated, and eviscerated. The spinal column was minced using sterile razor blades and trypsinized. A single-cell suspension was ensured by continuous mixing before PO cells were plated in monolayer culture. Dermal or renal primary fibroblasts were isolated from the PKD1 patient by skin biopsy or at nephrectomy and expanded by standard methods.

Statistics. All analyses were performed using JMP Pro9. For the murine studies, analyses were performed by 2-way ANOVA and least-squares means (LSMEANS) Student's $t$ post hoc test. A $P$ value below the $\alpha$ level of 0.05 was considered significant. A 2-tailed Student's $t$ test was used to compare means and SDs between densitometry-derived NTR and NTS relative band intensities. For cilia quantification, a 2-tailed Fisher's exact test was performed on data collected in contingency tables.

Study approval. The Mayo Clinic Institutional Animal Care and Utilization Committee approved the use of all murine models and experimental protocols within this study.

\section{Acknowledgments}

We thank several investigators for their gifts of models and reagents that made this study possible: Oxana Beskrovnaya for PC1 (CT) antibodies, Richard Sandford for the Pkd1 del17 model, 
Shigeo Horie for the $P k d 1$ del2 (null) model, Stefan Somlo for the Pkd2 del2 (null) and WS25 models, and Mahmoud LoghmanAdham for the RCTE and 9-12 cells. In addition, we thank William Wood, Jackie Sloane-Stanley, and Jackie Sharpe (Weatherall Institute of Molecular Medicine, University of Oxford, Oxford, United Kingdom) for assistance in generating the $P k d 1$ del31 model and Mathieu Mateo for helpful discussions and methods. The study was supported by NIDDK grant R01-DK058816 (to P.C. Harris), the NIDDK-funded Mayo Translational PKD Center (DK090728), Mayo Graduate School (to V.G. Gainullin), and an American Society of Nephrology, Ben J. Lipps Research Fellowship (to K. Hopp).

Address correspondence to: Peter C. Harris, Division of Nephrology and Hypertension, Mayo Clinic, 200 First Street SW, Rochester, Minnesota 55905, USA. Phone: 507.266.0541; E-mail: harris. peter@mayo.edu.
1. Torres VE, Harris PC, Pirson Y. Autosomal dominant polycystic kidney disease. Lancet. 2007;369(9569):1287-1301.

2. Mochizuki T, et al. PKD2, a gene for polycystic kidney disease that encodes an integral membrane protein. Science. 1996;272(5266):1339-1342.

3. Hughes J, et al. The polycystic kidney disease 1 (PKD1) gene encodes a novel protein with multiple cell recognition domains. Nat Genet. 1995;10(2):151-160.

4. Ravine D, et al. Phenotype and genotype heterogeneity in autosomal dominant polycystic kidney disease. Lancet. 1992;340(8831):1330-1333.

5. Cornec-Le Gall E, et al. Type of PKD1 mutation influences renal outcome in ADPKD. JAm Soc Nephrol. 2013;24(6):1006-1013.

6. Koulen P, et al. Polycystin-2 is an intracellular calcium release channel. Nat Cell Biol. 2002;4(3):191-197.

7. Gonzalez-Perrett S, et al. Polycystin-2, the protein mutated in autosomal dominant polycystic kidney disease (ADPKD), is a Ca2+-permeable non-selective cation channel. Proc Natl Acad Sci U S A. 2001;98(3):1182-1187.

8. Ponting CP, Hofmann K, Bork P. A latrophilin/ CL-1-like GPS domain in polycystin-1. Curr Biol. 1999;9(16):R585-R588.

9. Wei W, Hackmann K, Xu H, Germino G, Qian F. Characterization of cis-autoproteolysis of polycystin-1, the product of human polycystic kidney disease 1 gene. J Biol Chem. 2007;282(30):21729-21737.

10. Qian F, et al. Cleavage of polycystin-1 requires the receptor for egg jelly domain and is disrupted by human autosomal-dominant polycystic kidney disease 1- associated mutations. Proc Natl Acad Sci U S A. 2002;99(26):16981-16986.

11. Arac D, et al. A novel evolutionarily conserved domain of cell-adhesion GPCRs mediates autoproteolysis. EMBO J. 2012;31(6):1364-1378.

12. Yu S, et al. Essential role of cleavage of Polycystin-1 at G protein-coupled receptor proteolytic site for kidney tubular structure. Proc Natl Acad Sci U S A. 2007;104(47):18688-18693.

13. Cai $Y$, et al. Identification and characterization of polycystin-2, the PKD2 gene product. J Biol Chem. 1999;274(40):28557-28565.

14. Hogan MC, et al. Characterization of PKD protein-positive exosome-like vesicles. JAm Soc Nephrol. 2009;20(2):278-288.

15. Hanaoka K, et al. Co-assembly of polycystin-1 and-2 produces unique cation-permeable currents. Nature. 2000;408(6815):990-994.

16. Grimm DH, et al. Polycystin-1 distribution is modulated by polycystin-2 expression in mammalian cells. J Biol Chem. 2003;278(38):36786-36793.
17. Chapin HC, Rajendran V, Caplan MJ. Polycystin- 1 surface localization is stimulated by polycystin-2 and cleavage at the G protein-coupled receptor proteolytic site. Mol Biol Cell. 2010;21(24):4338-4348.

18. Newby LJ, Streets AJ, Zhao Y, Harris PC, Ward CJ, Ong ACM. Identification, characterization and localization of a novel kidney polycystin-1/polycystin-2 complex. J Biol Chem . 2002;277(23):20763-20773.

19. Hoffmeister H, et al. Polycystin-2 takes different routes to the somatic and ciliary plasma membrane. J Cell Biol. 2011;192(4):631-645.

20. Hopp K, et al. Functional polycystin-1 dosage governs autosomal dominant polycystic kidney disease severity. JClin Invest. 2012;122(11):4257-4273.

21. Hildebrandt F, Benzing T, Katsanis N. Ciliopathies. N Engl J Med. 2011;364(16):1533-1543.

22. Barr MM, Sternberg PW. A polycystic kidney-disease gene homologue required for male mating behaviour. Nature. 1999;401(6751):386-389.

23. Nauli SM, et al. Polycystins 1 and 2 mediate mechanosensation in the primary cilium of kidney cells. Nat Genet. 2003;33(2):129-137.

24. Haycraft CJ, Swoboda P, Taulman PD, Thomas $\mathrm{JH}$, Yoder BK. The C. elegans homolog of the murine cystic kidney disease gene $\mathrm{Tg} 737$ functions in a ciliogenic pathway and is disrupted in osm-5 mutant worms. Development 2001;128(9):1493-1505.

25. Pazour GJ, et al. Chlamydomonas IFT88 and its mouse homologue, polycystic kidney disease gene $\operatorname{Tg} 737$, are required for assembly of cilia and flagella. JCell Biol. 2000;151(3):709-718.

26. Yoder BK, Hou X, Guay-Woodford LM. The polycystic kidney disease proteins, polycystin-1, polycystin-2, polaris, and cystin, are co-localized in renal cilia. JAm Soc Nephrol. 2002;13(10):2508-2516.

27. Pazour GJ, San Agustin JT, Follit JA, Rosenbaum JL, Witman GB. Polycystin-2 localizes to kidney cilia and the ciliary level is elevated in orpk mice with polycystic kidney disease. Curr Biol. 2002;12(11):R378-R380.

28. Ishikawa H, Thompson J, Yates JR 3rd, Marshall WF. Proteomic analysis of mammalian primary cilia. Curr Biol. 2012;22(5):414-419.

29. Delling M, DeCaen PG, Doerner JF, Febvay S, Clapham DE. Primary cilia are specialized calcium signalling organelles. Nature. 2013;504(7479):311-314.

30. Ma M, Tian X, Igarashi P, Pazour GJ, Somlo S. Loss of cilia suppresses cyst growth in genetic models of autosomal dominant polycystic kidney disease. Nat Genet. 2013;45(9):1004-1012.

31. Qian F, Germino FJ, Cai Y, Zhang X, Somlo S,
Germino GG. PKD1 interacts with PKD2 through a probable coiled-coil domain. Nat Genet. 1997;16(2):179-183.

32. Tsiokas L, Kim E, Arnould T, Sukhatme VP, Walz G. Homo- and heterodimeric interactions between the gene products of PKD1 and PKD2. Proc Natl Acad Sci U S A. 1997;94(13):6965-6970.

33. Celic A, Petri ET, Demeler B, Ehrlich BE, Boggon TJ. Domain mapping of the polycystin-2 C-terminal tail using de novo molecular modeling and biophysical analysis. J Biol Chem. 2008;283(42):28305-28312.

34. Giamarchi A, et al. A polycystin-2 (TRPP2) dimerization domain essential for the function of heteromeric polycystin complexes. EMBO J. 2010;29(7):1176-1191.

35 . Geng L, et al. Polycystin-2 traffics to cilia independently of polycystin-1 by using an N-termina RVxP motif. JCell Sci. 2006;119(pt 7):1383-1395.

36. Yu Y, et al. Structural and molecular basis of the assembly of the TRPP2/PKD1 complex. Proc Natl Acad Sci U S A. 2009;106(28):11558-11563.

37. Sharif-Naeini R, et al. Polycystin-1 and -2 dosage regulates pressure sensing. Cell. 2009;139(3):587-596.

38. Tsiokas L, Arnould T, Shu C, Kim E, Walz G, Sukhatme VP. Specific association of the gene product of PKD2 with the TRPC1 channel. Proc Natl Acad Sci U S A. 1999;96(7):3934-3939.

39. Kottgen M, et al. TRPP2 and TRPV4 form a polymodal sensory channel complex. JCell Biol. 2008;182(3):437-447.

40. McGrath J, Somlo S, Makova S, Tian X, Brueckner M. Two populations of node monocilia initiate left-right asymmetry in the mouse. Cell. 2003;114(1):61-73.

41. Karcher C, et al. Lack of a laterality phenotype in Pkd1 knock-out embryos correlates with absence of polycystin-1 in nodal cilia. Differentiation. 2005;73(8):425-432.

42. Field S, et al. Pkd1l1 establishes left-right asymmetry and physically interacts with $\mathrm{Pkd} 2$. Development. 2011;138(6):1131-1142.

43. Lu WN, et al. Perinatal lethality with kidney and pancreas defects in mice with a targetted Pkd1 mutation. Nat Genet. 1997;17(2):179-181.

44. Lu W, et al. Late onset of renal and hepatic cysts in Pkd1-targeted heterozygotes. Nat Genet. 1999;21(2):160-161.

45. Wu G, et al. Cardiac defects and renal failure in mice with targeted mutations in Pkd2. Nat Genet. 2000;24(1):75-78.

46. Wu G, et al. Trans-heterozygous Pkd1 and Pkd2 mutations modify expression of polycystic kidney disease. Hum Mol Genet. 2002;11(16):1845-1854. 47. Pei Y, et al. Bilineal disease and trans-heterozy- 
gotes in autosomal dominant polycystic kidney disease. Am J Hum Genet. 2001;68(2):355-363.

48. Pei Y, et al. A missense mutation in PKD1 attenuates the severity of renal disease. Kidney Int. 2012;81(4):412-417.

49. Gallagher AR, Germino GG, Somlo S. Molecular advances in autosomal dominant polycystic kidney disease. Adv Chronic Kidney Dis. 2010;17(2):118-130.

50. Lantinga-van Leeuwen IS, et al. Lowering of Pkd1 expression is sufficient to cause polycystic kidney disease. Hum Mol Genet. 2004;13(24):3069-3077.

51. Ward CJ, et al. Polycystin, the polycystic kidney disease 1 protein, is expressed by epithelial cells in fetal, adult and polycystic kidney. Proc Natl Acad Sci U S A. 1996;93(4):1524-1528.

52. Elbein AD, Szumilo T, Sanford BA, Sharpless KB, Adams C. Effect of isomers of swainsonine on glycosidase activity and glycoprotein processing. Biochemistry. 1987;26(9):2502-2510.

53. Varki A. Glycan-based interactions involving vertebrate sialic-acid-recognizing proteins. Nature. 2007;446(7139):1023-1029.

54. Igarashi P, Somlo S. Genetics and pathogenesis of polycystic kidney disease. J Am Soc Nephrol. 2002;13(9):2384-2398.

55. Park CY, et al. STIM1 clusters and activates CRAC channels via direct binding of a cytosolic domain to Orai1. Cell. 2009;136(5):876-890.

56. Yeromin AV, Zhang SL, Jiang W, Yu Y, Safrina
O, Cahalan MD. Molecular identification of the CRAC channel by altered ion selectivity in a mutant of Orai. Nature. 2006;443(7108):226-229.

57. Hopp K, Hommerding CJ, Wang X, Ye H, Harris PC, Torres VE. Tolvaptan plus pasireotide shows enhanced efficacy in a PKD1 model [published online ahead of print July 3, 2014]. J Am Soc Nephrol. doi:ASN.2013121312.

58. Wu G, et al. Somatic inactivation of Pkd2 results in polycystic kidney disease. Cell. 1998;93(2):177-188.

59. Boulter C, Mulroy S, Webb S, Fleming S, Brindle K, Sandford R. Cardiovascular, skeletal, and renal defects in mice with a targeted disruption of the Pkd1 gene. Proc Natl Acad Sci U S A. 2001;98(21):12174-12179.

60. [No authors listed]. The polycystic kidney disease 1 gene encodes a $14 \mathrm{~kb}$ transcript and lies within a duplicated region on chromosome 16. Cell. 1994;77(6):881-894.

61. Rossetti S, et al. Comprehensive molecular diagnostics in autosomal dominant polycystic kidney disease. J Am Soc Nephrol. 2007;18(7):2143-2160.

62. Freedman BS, et al. Reduced ciliary polycystin-2 in induced pluripotent stem cells from polycystic kidney disease patients with PKD1 mutations. JAm Soc Nephrol. 2013;24(10):1571-1586.

63. Kurbegovic A, et al. Novel functional complexity of polycystin- 1 by GPS cleavage in vivo: role in polycystic kidney disease. Mol Biol Cell. 2014;34(17):3341-3353.
64. Oatley P, Talukder MMU, Stewart AP, Sandford R, Edwardson JM. Polycystin-2 induces a conformational change in polycystin-1. Biochemistry. 2013;52(31):5280-5287.

65. Watnick T, et al. Mutations of PKD1 in ADPKD2 cysts suggest a pathogenic effect of trans-heterozygous mutations. Nat Genet. 2000;25(2):143-144.

66. Koptides M, Mean R, Demetriou K, Pierides A, Deltas CC. Genetic evidence for a trans-heterozygous model for cystogenesis in autosomal dominant polycystic kidney disease. Hum Mol Genet. 2000;9(3):447-452.

67. Zeng Y, Ramya TN, Dirksen A, Dawson PE, Paulson JC. High-efficiency labeling of sialylated glycoproteins on living cells. Nat Methods. 2009;6(3):207-209.

68. Nauli SM, et al. Loss of polycystin-1 in human cyst-lining epithelia leads to ciliary dysfunction. JAm Soc Nephrol. 2006;17(4):1015-1025.

69. Muto S, et al. Pioglitazone treatment improved the cardiac and renal cystic phenotype of a targeted Pkd1 mutant. Hum Mol Genet. 2002;11(15):1731-1742.

70. Ong AC, et al. Polycystin-1 expression in PKD1, early-onset PKD1, and TSC2/PKD1 cystic tissue. Kidney Int. 1999;56(4):1324-1333.

71. Ward CJ, et al. Polycystin, the polycystic kidney disease 1 protein, is expressed by epithelial cells in fetal, adult, and polycystic kidney. Proc Natl Acad Sci U S A. 1996;93(4):1524-1528. 